FTPI-MINN-05/07

UMN-TH-2348/05

hep-ph/0505029

\title{
Lorentz Violating Supersymmetric Quantum Electrodynamics
}

\author{
Pavel A. Bolokhov ${ }^{1,3}$, Stefan Groot Nibbelink ${ }^{2}$ and Maxim Pospelov Pr, $^{1,4}$ \\ ${ }^{1}$ Department of Physics and Astronomy, University of Victoria, Victoria, \\ $B C$, V8P 1A1, Canada \\ ${ }^{2}$ William I. Fine Theoretical Physics Institute, University of Minnesota, \\ Minneapolis, MN 55455, USA \\ ${ }^{3}$ Theoretical Physics Department, St. Petersburg State University, Ulyanovskaya 1, \\ Peterhof, St. Petersburg, 198504, Russia \\ ${ }^{4}$ Perimeter Institute, 31 Caroline Street North, Waterloo, ON, N2J 2W9, Canada \\ ${ }^{5}$ Department of Physics, University of Guelph, Guelph, ON, N1G 2W1, Canada
}

\begin{abstract}
The theory of Supersymmetric Quantum Electrodynamics is extended by interactions with external vector and tensor backgrounds, that are assumed to be generated by some Lorentz-violating (LV) dynamics at an ultraviolet scale perhaps related to the Planck scale. Exact supersymmetry requires that such interactions correspond to LV operators of dimension five or higher, providing a solution to the naturalness problem in the LV sector. We classify all dimension five and six LV operators, analyze their properties at the quantum level and describe observational consequences of LV in this theory. We show that LV operators do not induce destabilizing D-terms, gauge anomaly and the Chern-Simons term for photons. We calculate the renormalization group evolution of dimension five LV operators and their mixing with dimension three LV operators, controlled by the scale of the soft-breaking masses. Dimension five LV operators are constrained by low-energy precision measurements at $10^{-10}-10^{-5}$ level in units of the inverse Planck scale, while the Planck-scale suppressed dimension six LV operators are allowed by observational data.
\end{abstract}




\section{INTRODUCTION}

There are many known examples in the history of physics when a symmetry of nature, which was assumed to be exact, has fallen under experimental scrutiny. The study of the consequences of such breaking has often provided important insights into the dynamics at high-energy scales. This was exemplified by the weak-scale dynamics of the Standard Model (SM) through the search and discovery of $\mathrm{P}$ and $\mathrm{CP}$ violations. Lorentz symmetry is used as a crucial ingredient in the construction of fundamental theories of nature. Even though no breakdown of this symmetry has been observed to date, there has been a growing interest in Lorentz Violation (LV) because the precision tests of Lorentz symmetry can provide an important window into the physics far beyond the electroweak scale [1, 2, [3, 4, [5, 6].

The recently intensified interest in LV theories is stimulated by several seemingly unrelated motives. Firstly, a combination of different sets of cosmological data indicates that the dominant component of the energy density of the universe is dark energy. It can either be ascribed to a cosmological constant or to an energy density associated with a new infrared degree of freedom, such as e.g. an ultra-light scalar field (quintessence). The time evolution of quintessence creates a preferred frame, which could in principle be detected as a LV background, provided that it couples to the SM. Secondly, low-energy limits of string theory contain a number of (nearly) massless fields, some of which carry open Lorentz indices. The well-studied example of an antisymmetric field background $B_{\mu \nu}$ on a brane (for a review see [7]) leads to an effective violation of Lorentz invariance. Thirdly, there have been a number of conjectures that a theory of quantum gravity could manifest itself at lower energies through LV modifications of particle dispersion relations (see, e.g. 8, 9] and references therein). Although such conjectures are undoubtedly speculative, if true, they would provide a powerful tool to probe ultra-short distances via LV physics. Direct experimental constraints on modifications of dispersion relations come from astrophysical processes 10, 11, 12, 13, 14, 15, 16, 17] and terrestrial clock comparison experiments [18, 19, 20, 21]. In both cases the typical sensitivity to these operators is at the $10^{-5} / M_{\mathrm{Pl}}$ level. This creates a definite problem for those theories that predict Planck mass suppressed $\sim 1 / M_{\mathrm{Pl}}$ effects.

In an effective field theory framework, the breakdown of Lorentz symmetry can be described by the presence of external tensors, which are generated by some unspecified dynamics, coupled to SM operators. It is useful to characterize such operators by powers of 
increasing dimension, as it gives an indication to the possible scaling of LV effects with the ultraviolet (UV) scale $M$, which might be related to $M_{\mathrm{Pl}}$. In quantum electrodynamics (QED), the generic expansion in terms of the gauge invariant operators starts at dimension three (see e.g. [1]):

$$
\mathcal{L}_{\mathrm{QED}}^{(3)}=-a_{\mu} \bar{\Psi} \gamma_{\mu} \Psi-b_{\mu} \bar{\Psi} \gamma^{\mu} \gamma_{5} \Psi-\frac{1}{2} H_{\mu \nu} \bar{\Psi} \sigma^{\mu \nu} \Psi-k_{\mu} \epsilon^{\mu \nu \kappa \lambda} A_{\nu} \partial_{\kappa} A_{\lambda} .
$$

Here $\psi$ is a Dirac spinor describing the electron and $A_{\mu}$ is the electromagnetic vectorpotential. The external vector and anti-symmetric tensor backgrounds, $a_{\mu}, b_{\mu}, k_{\mu}$ and $H_{\mu \nu}$ define a preferred frame, and therefore break Lorentz invariance. The coupling to the vector current, $a_{\mu} \bar{\Psi} \gamma^{\mu} \Psi$, can be removed by introducing a space-time dependent phase for the electron. The last term in the Lagrangian (1), the Chern-Simons term, is gauge invariant up to a total derivative, which can be neglected.

At this dimension three level there is a problem in ascribing LV to UV dynamics. From simple dimensional counting one would expect the external vectors and tensors, $a_{\mu}, b_{\mu}, \ldots$ to be on the order of the UV scale $M$, and therefore LV would be very large, which is clearly inadmissible. For example, a Higgs mechanism resulting in a condensation of a vector field $V_{\mu} \sim M n_{\mu}$ (where $n_{\mu}$ is a "unit" vector [22]) creates disastrous consequences when coupled to a non-conserved current, i.e. the axial current $\bar{\Psi} \gamma_{\mu} \gamma_{5} \Psi$. One may hope that operators of dimension three and four are forbidden by some symmetry arguments or tuned to be small, so that LV effects first appear at dimension five (or higher) level 21]. However, such hopes are typically shattered by quantum corrections, which lead to dimensional transmutation of a higher dimensional operator into a lower dimensional one with a quadratically divergent coefficient:

$$
[L V]_{\operatorname{dim} 3} \sim \text { (loop factor) } \Lambda_{U V}^{2} \times[L V]_{\operatorname{dim} 5} .
$$

Here $[L V]_{\operatorname{dim} 3(5)}$ represent generic LV operators of dimension three and five, respectively. If the UV cutoff scale $\Lambda_{U V}$ is of the order of $M$, huge dimension three operators are generated. In that case all higher dimensional operators would have to be tuned, leaving no room for LV interactions. This naturalness problem of LV physics can be avoided if these quadratic divergences are suppressed by certain symmetry arguments. In Ref. [21] it was shown that dimension five LV operators coupled to fully symmetric three-index traceless tensors are protected against developing quadratic loop divergences. But this solves the naturalness problem only partially, as this does not provide an argument as to why dimension three and 
four operators cannot be induced at tree level, and why they have to be tuned by hand to experimentally acceptable values.

A recent paper 23] proposed that supersymmetry (SUSY) could provide a powerful selection rule on admissible forms of LV interactions. In particular, it has been shown that in the Minimal Supersymmetric Standard Model (MSSM) the requirements of SUSY and gauge invariance restrict LV operators to be of dimension five or higher. Therefore SUSY solves the naturalness problem of LV physics. Once SUSY is softly broken, the quadratic UV divergences are effectively stabilized at the supersymmetric threshold. Hence this might lead to a solution of the question of why the lower dimensional LV operators are so much suppressed as compared to their natural scale.

An explicit example of how SUSY restricts possible LV interactions and leads to dramatic numerical changes in the predicted observables is provided by non-commutative field theories. The non-commutative background tensor $\theta_{\mu \nu}$, entering the Moyal product, has the canonical dimension -2 , and therefore the scale of non-commutativity, $\Lambda_{N C} \sim(\theta)^{-1 / 2}$, gives a natural UV scale. As a result, linearizing the action in $\theta$ is justified, as long as the characteristic momenta are much smaller than $\Lambda_{N C}$. This expansion leads to a set of dimension six operators, which at the tree level induce interactions between particle spins and the $\theta_{\mu \nu}$ background [24] with effective $H_{\mu \nu}$ in (11) given by $H_{\mu \nu} \sim \Lambda_{I R}^{3} \theta_{\mu \nu}$. Here $\Lambda_{I R}$ is the relevant infrared scale, such as $\Lambda_{Q C D}$ in the case of hadrons. However, it has been shown that loop effects in non-commutative field theories lead to quadratically divergent integrals [25]: $H_{\mu \nu} \sim \Lambda_{I R} \Lambda_{U V}^{2} \theta_{\mu \nu}$. This essentially invalidates the expansion in terms of $\theta_{\mu \nu}$. If the cutoff scale is very high, e.g. comparable to $\Lambda_{N C}$, the resulting spin anisotropy is large and certainly excluded by experiment. However, this conclusion is premature as one can argue that the operator $\bar{q} \sigma_{\mu \nu} q$ is incompatible with SUSY [26] and thus should not be induced in the domain of the loop momenta higher than the SUSY breaking. This means that the cutoff essentially coincides with the energy splitting between fermions and bosons, i.e. $\Lambda_{U V} \sim m_{\text {soft }}$. This has been confirmed by an explicit two-loop calculation in the framework of non-commutative supersymmetric QED [27]. With the quadratic divergences stabilized at $m_{\text {soft }} \sim 1 \mathrm{TeV}$, the Planck scale non-commutativity is safely within the experimental bounds. This example illustrates that the existence of SUSY can be important for understanding the actual size of the expected LV effects. Another example as to how SUSY can protect against quadratic divergences in a LV theory has been given recently in [28]. 
The purpose of this work is to analyze in detail LV operators in supersymmetric quantum electrodynamics (SQED), to prove the absence of the naturalness problem in the LV sector, and to derive phenomenological constraints on the LV parameters in SQED. We see this as a first step towards the phenomenological analysis of the full LV MSSM. Following Ref. [23], we parametrize all dimension five operators of LV SQED by three vector $N^{\mu}, N_{+}^{\mu}$ and $N_{-}^{\mu}$ and one irreducible rank three tensor $T^{\mu \nu \lambda}$ backgrounds. These vector and tensor backgrounds enter in the LV operators composed of a vector superfield (containing photons and photinos) and chiral superfields (corresponding to left- and right-handed (s)electrons), respectively. We introduce these CPT-violating operators in the superfield formalism, and then derive their component form. We also classify dimension six CPT-conserving LV operators in superspace notations. We observe that by using the equations of motion (EOM's) some parts of dimension five operators can be reduced to dimension three LV operators. The relation between them $[L V]_{\operatorname{dim} 3} \sim m_{e}^{2}[L V]_{\operatorname{dim} 5}$ is controlled by the electron mass $m_{e}$.

The main emphasis of our study is on the quantum effects. We show that even in the presence of SUSY LV operators no destabilizing quadratically divergent D-terms ever arise. We prove that gauge anomalies are not affected by the presence of these LV operators. This analysis essentially implies that the Chern-Simons (CS) term cannot arise from quantum corrections. We derive the renormalization group ( $R G)$ evolution for the LV operators, showing explicitly that only the logarithmic divergences arise in the limit of exact SUSY. We solve the one-loop renormalization group equations (RGE's) to obtain the low-energy values of LV parameters in terms of their values at the UV scale $M$. Then we investigate the consequences of SUSY breaking for LV operators by introducing the soft-breaking masses for superpartners of electrons. Dimension three LV operators can now be induced by dimensional transmutation: $[L V]_{\operatorname{dim} 3} \sim m_{\text {soft }}^{2}[L V]_{\operatorname{dim} 5}$. Although a loop effect, this constitutes a dramatic enhancement compared to the case with unbroken SUSY, as $m_{\text {soft }}^{2} / m_{e}^{2}>10^{10}$. One might expect that similar quantum corrections could induce a CS term (the last operator in (II)) once SUSY is broken. However, our analysis rules out this possibility.

We investigate phenomenological consequences of LV in the framework of softly-broken SQED. The strongest constraints on the LV parameters are due to the (non)observation of anomalous spin precession around directions defined by the LV background vectors $N^{\mu}, N_{+}^{\mu}$ and $N_{-}^{\mu}$. Another constraint comes from the comparison of the anomalous magnetic moments of electrons and positrons. It is important to note that all constraints obtained in this work 
are laboratory constraints, as astrophysical and cosmological searches of LV are not sensitive to LV effects in SQED.

We present our results in the following order. Section $\amalg$ introduces the LV operators and backgrounds at dimension five and six levels. Section [II investigates various quantum corrections to LV operators under the assumption of exact SUSY. Subsection IIA shows that no dangerous quadratically divergent D-terms arise. Subsection Щ1 explains that no novel gauge anomalies can ever appear due to LV operators, and consequently that a SUSY CS term is ruled out. Finally, subsection IIC addresses the running of LV operators of dimension five. Section [V studies the consequences of soft SUSY breakdown for the LV sector, and derives RGE's for the induced dimension three LV operators. In subsection IVB we argue that even when SUSY is broken no CS term is generated. In section $\nabla$ we study the phenomenology of the model, and obtain various predictions for relevant LV observables. We reach our conclusions in section VI.

\section{LV OPERATORS IN SQED}

Supersymmetric Quantum Electrodynamics (SQED) is described by two chiral superfields $\Phi_{+}$and $\Phi_{-}$, that are oppositely charged under a $\mathrm{U}(1)$ gauge superfield $V$ :

$$
\begin{aligned}
\mathcal{L}_{\mathrm{S} Q E D}= & \int d^{4} \theta\left(\bar{\Phi}_{+} e^{2 e V} \Phi_{+}+\bar{\Phi}_{-} e^{-2 e V} \Phi_{-}\right) \\
& +\int d^{2} \theta\left(\frac{1}{4} W W+m_{e} \Phi_{-} \Phi_{+}\right)+\int d^{2} \bar{\theta}\left(\frac{1}{4} \bar{W} \bar{W}+\bar{m}_{e} \bar{\Phi}_{+} \bar{\Phi}_{-}\right) .
\end{aligned}
$$

Here $W_{\alpha}=-\frac{1}{4} \bar{D}^{2} D_{\alpha} V$ is the super gauge invariant expression for the field strength. Throughout this paper, we use predominantly Wess and Bagger notations 29]. The fermionic components of superfields $\Phi_{+}$and $\Phi_{-}$correspond to the left-handed electron and righthanded charge-conjugated electron fields. With a slight abuse of the language, we call them the electron and positron superfields, or just the electron and the positron for brevity. We define the charge of electron as $e=-|e|$. Finally, $m_{e}$ denotes the (complex) electron mass.

LV extensions of SQED can be constructed as a set of effective operators containing the superfields $\Phi_{-}, \Phi_{+}$, gauge covariant derivatives $\nabla_{\alpha}, \bar{\nabla}_{\dot{\alpha}}$ and arbitrary constant tensor coefficients with Lorentz indices that specify the breakdown of Lorentz invariance [23]. The general rules according to which LV operators should be constructed are listed in Ref. [21]. 
Within the context of SQED, however, we impose additional requirements related to supersymmetry. In this work we require that all LV operators be

- supersymmetric,

- local super gauge invariant with chiral gauge parameters,

- have local component expressions.

Let us explain these conditions in more detail.

First of all, by having supersymmetry we mean that the sub-algebra

$$
\left\{Q_{\alpha}, \bar{Q}_{\dot{\alpha}}\right\}=2 \sigma_{\alpha \dot{\alpha}}^{\mu} P_{\mu}
$$

of the $N=1$ super Poincaré algebra remains unbroken. (LV Theories with higher amounts of SUSY coming from extra dimensions have also been investigated [30, 31, 32]). If we assume that the breaking of the Lorentz symmetry is spontaneous, we are guaranteed that $\sigma_{\alpha \dot{\alpha}}^{\mu}$ represent the standard Pauli matrices. However, if the breaking of Lorentz symmetry is explicit from the outset of the theory, these objects are simply structure coefficients parameterizing this supersymmetry algebra. (In this work we do not pursue this possibility further. Possible modifications of superalgebra by LV parameters has been discussed in Ref. [33, 34].) This assumption allows us to perform our analysis using conventional superspace.

The requirement of having a local component expression allows for a conventional effective field theory interpretation of the Lagrangians that we obtain. However, the locality of the component Lagrangian does not necessarily imply that the superspace expression of a given Lagrangian appears local [56]. For example, the electron mass term can be written in a seemingly non-local way: $\int d^{4} \theta m_{e} \Phi_{-} D^{2} /(-4 \square) \Phi_{+}+$h.c.. Finally, we require that LV operators preserve the standard local super gauge transformations

$$
\Phi_{ \pm} \rightarrow e^{\mp 2 e \Lambda} \Phi_{ \pm}, \quad \bar{\Phi}_{ \pm} \rightarrow e^{\mp 2 e \bar{\Lambda}} \bar{\Phi}_{ \pm}, \quad V \rightarrow \Lambda+\bar{\Lambda},
$$

with a chiral parameter $\Lambda$. In particular, we do not allow for non-local or non-chiral extensions of the gauge transformations that seem to be required by non-commutative SUSY [35, 36].

As was shown in [23], these conditions combined impose strong restrictions on the number of LV terms of a specific mass dimension one can construct: no dimension three or four LV 
operators can be written down within the context of the MSSM. Here we do not repeat all the arguments leading to this general claim, but simply illustrate the underlying philosophy by showing that the CS term (the last interaction in (10)) does not have a SUSY extension satisfying all three conditions stated above.

The CS term is a dimension three operator that is bilinear in the gauge field and proportional to an external vector. Therefore the local superspace extension of it can be represented as

$$
\mathcal{L}_{\mathrm{SCS}}^{\text {local }}=\frac{1}{2} k^{\mu} \int d^{4} \theta \bar{\sigma}_{\mu}^{\dot{\alpha} \alpha} V\left[D_{\alpha}, \bar{D}_{\dot{\alpha}}\right] V=k_{\mu}\left(-\epsilon^{\mu \lambda \nu \rho} A_{\lambda} \partial_{\nu} A_{\rho}+2 A^{\mu} D+2 \bar{\lambda} \bar{\sigma}^{\mu} \lambda\right) .
$$

This is the only possible structure, as the insertion of an anti-commutator $\left\{D_{\alpha}, \bar{D}_{\dot{\alpha}}\right\}$ immediately gives rise to a total spacetime derivative. The component expression shows that this operator indeed contains the CS term, which is gauge invariant up to a total derivative. However, the SUSY extension as a whole is not super gauge invariant:

$$
\delta \mathcal{L}_{\mathrm{SCS}}^{\text {local }}=2 i k^{\mu} \int d^{4} \theta V \partial_{\mu}(\bar{\Lambda}-\Lambda)
$$

Notice that this statement is independent of the Wess-Zumino gauge, and that even under the restriction of gauge invariance under ordinary $\mathrm{U}(1)$ transformations $(\Lambda=i \alpha)$ the supersymmetric extension (and the $A^{\mu} D$ term in particular) of the CS term fails to be gauge invariant.

These arguments do not show that it is impossible to construct a super gauge invariant extension of the CS term. Indeed, by inserting the transversal projector $P_{V}=D^{\alpha} \bar{D}^{2} D_{\alpha} /(-8 \square)$ we obtain a manifestly super gauge invariant expression

$$
\mathcal{L}_{\mathrm{SCS}}^{\text {non-local }}=\frac{1}{2} k^{\mu} \int d^{4} \theta \bar{\sigma}_{\mu}^{\dot{\alpha} \alpha} V P_{V}\left[D_{\alpha}, \bar{D}_{\dot{\alpha}}\right] V=k^{\mu} \int d^{4} \theta \bar{W} \bar{\sigma}_{\mu} \frac{1}{\square} W .
$$

This expression clearly appears to be non-local in superspace, but the CS term itself is still local. In fact, because the true CS term in (6) already was gauge invariant, the insertion of $P_{V}$ did not affect it at all. However, other terms in the component expression of (8) are non-local because they contain $1 / \square$ explicitly. Hence, as asserted, the CS term does not allow for a SUSY extension that is super gauge invariant and that has a local component expression. Additional discussion of LV due to a CS term in supersymmetric theories can be found in Refs. [37, 38]. 


\section{A. CPT-violating dimension five LV operators}

There are only three different types of LV operators satisfying the above requirements in SQED at the dimension five level. In this subsection we give their superfield expressions, while their component forms can be found in Section $\square$ The first type is the electron and positron superfield operators

$$
\mathcal{L}_{\mathrm{LV}}^{\text {matter }}=\frac{1}{M} \int d^{4} \theta\left\{N_{+}^{\mu} \bar{\Phi}_{+} e^{2 e V} i \nabla_{\mu} \Phi_{+}+N_{-}^{\mu} \bar{\Phi}_{-} e^{-2 e V} i \nabla_{\mu} \Phi_{-}\right\},
$$

which are parameterized by two external real vectors $N_{ \pm}^{\mu}$. The super gauge covariant spacetime derivative $\nabla_{\mu}=-\frac{i}{4} \bar{\sigma}_{\mu}^{\dot{\alpha} \alpha}\left\{\nabla_{\alpha}, \bar{\nabla}_{\dot{\alpha}}\right\}$ is defined in terms of the super gauge covariant derivatives $\nabla_{\alpha}$ and $\bar{\nabla}_{\dot{\alpha}}$. Their precise form depends on the super gauge transformation properties of the object that they act on. For example, we define

$$
\nabla_{\alpha} \Psi_{ \pm}=e^{\mp 2 e V} D_{\alpha}\left(e^{ \pm 2 e V} \Psi_{ \pm}\right), \quad \bar{\nabla}_{\dot{\alpha}} \Psi_{ \pm}=\bar{D}_{\dot{\alpha}} \Psi_{ \pm}
$$

for generic superfields $\Psi_{ \pm}$, that have the same gauge transformations as the (chiral) superfields $\Phi_{ \pm}$, see (515).

For the photon super multiplet we can construct two independent operators. The first operator is parameterized by a real vector $N^{\mu}$. We can give a Kähler-like representation of this vector operator as

$$
\mathcal{L}_{\mathrm{LV} \mathrm{dim} 5}^{\text {gauge }(\mathrm{V})}=\frac{1}{M} \int d^{4} \theta N^{\kappa} \bar{W} \bar{\sigma}_{\kappa} W
$$

Using a superspace identity, this operator can also be written as a superpotential-like term

$$
\mathcal{L}_{\mathrm{LV} \text { dim } 5}^{\text {gauge }(\mathrm{V})}=-\frac{N_{\kappa}}{2 M} \epsilon^{\kappa \lambda \mu \nu}\left(\int d^{2} \theta W \sigma_{\mu \nu} \partial_{\lambda} W+\int d^{2} \bar{\theta} \bar{W} \bar{\sigma}_{\mu \nu} \partial_{\lambda} \bar{W}\right) .
$$

The most general LV superpotential-like term takes the form

$$
\mathcal{L}_{\mathrm{LV} \text { dim } 5}^{\text {gauge (T) }}=\frac{1}{4 M} \int d^{2} \theta T^{\lambda \mu \nu} W \sigma_{\mu \nu} \partial_{\lambda} W+\frac{1}{4 M} \int d^{2} \theta \bar{T}^{\lambda \mu \nu} \bar{W} \bar{\sigma}_{\mu \nu} \partial_{\lambda} \bar{W} .
$$

In principle this operator is parameterized by a complex rank-three tensor $T^{\lambda \mu \nu}$, antisymmetric in the last two indices $(\mu, \nu)$ due to its contraction with $\sigma_{\mu \nu}$. Notice that $\sigma_{\mu \nu}$ acts as a projector on the imaginary self-dual part of the tensor since $\frac{1}{2} i \epsilon_{\mu \nu}{ }^{\rho \sigma} \sigma_{\rho \sigma}=\sigma_{\mu \nu}$. This implies that we may take $T^{\lambda \mu \nu}$ real. We can constrain it further by requiring that

$$
T_{\mu}^{\mu \rho}=0, \quad \epsilon_{\kappa \lambda \rho \sigma} T^{\lambda \rho \sigma}=0 .
$$


The first condition arises because any trace part of $T^{\lambda \mu \nu}$ of the operator (13) vanishes, as $\int d^{2} \theta W \sigma^{\mu \nu} \partial_{\nu} W+\int d^{2} \theta \bar{W} \bar{\sigma}^{\mu \nu} \partial_{\nu} \bar{W}=0$. The second condition ensures that the LV due to a vector background is entirely accounted for by (12).

In a non-Abelian theory, operator (13) cannot exist because the $W_{\alpha}$ 's are not gauge invariant but only gauge covariant. Thus, to maintain gauge invariance, any derivative acting on $W_{\alpha}$ has to be replaced by a corresponding super gauge derivative. In particular, a non-Abelian generalization of (13) would have to contain the covariant derivative $\nabla_{\mu}$. But then the integrand would not be chiral, as

$$
\bar{D}_{\dot{\gamma}} \nabla_{\mu} W_{\alpha}=-i e\left(\epsilon \bar{\sigma}_{\mu}\right)_{\dot{\gamma}}^{\beta}\left(W_{\alpha} W_{\beta}+W_{\beta} W_{\alpha}\right) \neq 0
$$

Therefore, in the non-Abelian case one cannot write down superpotential-like LV terms for gauge multiplets, and only the Kähler-like terms (11) are allowed.

We have listed all possible dimension five operators in SQED framework. These results have been reported before in the MSSM setting [23]. All operators of dimension 5, listed in this section, break CPT invariance. The CPT-conserving LV operators start at dimension 6 level. For the matter of completeness, we now classify all dimension six LV operators compatible with SQED. However, our main analysis of quantum loop effects and observational implications of LV will be concentrated on the dimension five operators (9), (11) and (13).

\section{B. CPT-conserving dimension six LV operators}

Let us start by considering possible superpotential-like terms. To obtain dimension six operators in the Lagrangian density, one has to consider the superpotential at dimension five level, i.e. two dimensions higher than the standard mass term $m_{e} \Phi_{-} \Phi_{+}$. Because of the chirality condition of the superpotential, gauge invariance and the absence of fermionic LV backgrounds, all possible terms have to be built out of the (dimension two) operator $\Phi_{+} \Phi_{-}$ and the (dimension three) operator $W_{\alpha} W_{\beta}$, with possible derivative insertions in the latter. Omitting all Lorentz-preserving terms in the superpotential, we arrive at the following LV operator at dimension six level,

$$
\mathcal{L}_{\mathrm{LV} \operatorname{dim} 6}^{\text {super }}=\frac{1}{M^{2}} \int d^{2} \theta S^{\mu \nu} W \partial_{\mu} \partial_{\nu} W+\text { h.c. } .
$$

The dimensionless matrix $S$ is symmetric: $S^{\mu \nu}=S^{\nu \mu}$. All other possible operators would involve $W \sigma_{\mu \nu} W$ which vanishes for a single $\mathrm{U}(1)$. As mentioned before, the superpotential 
term (16) can be represented as an integral over the full superspace by factoring out $-\frac{1}{4} \bar{D}^{2}$. This can be done in various ways leading to seemingly different expressions for these operators. Since the superpotential expression above defines these operators uniquely, there is no need to give full superspace representations of these operators here.

Aside from the operator (16), we can construct gauge invariant LV operators from the (dimension two) building blocks $\bar{\Phi}_{ \pm} e^{ \pm 2 e V} \Phi_{ \pm}, \Phi_{-} \Phi_{+}, \bar{\Phi}_{-} \bar{\Phi}_{+}, D_{\alpha} W_{\beta}$ and $\bar{D}_{\dot{\alpha}} \bar{W}_{\dot{\beta}}$ with possible gauge covariant derivatives inserted. From the identity $\left[\nabla_{\mu}, \nabla_{\nu}\right] \Phi_{ \pm}= \pm e\left(\epsilon^{T} \sigma\right)^{\alpha \beta} \nabla_{\alpha}\left(W_{\beta} \Phi_{ \pm}\right)$ and the anti-chirality of $\bar{\Phi}_{ \pm}$, we infer that

$$
\int d^{4} \theta \bar{\Phi}_{ \pm} e^{ \pm 2 e V}\left[\nabla_{\mu}, \nabla_{\nu}\right] \Phi_{ \pm}=\int d^{4} \theta \Phi_{-}\left[\nabla_{\mu}, \nabla_{\nu}\right] \Phi_{+}=0
$$

Moreover, full superspace integrals of $\Phi_{-} \Phi_{+} D_{\alpha} W_{\beta}, \Phi_{-} \Phi_{+} \bar{D}_{\dot{\alpha}} \bar{W}_{\dot{\beta}}$ and their conjugates vanish as well. Therefore, the most general (genuine Kähler and non-reducible to superpotential) dimension six LV matter Lagrangian is given by

$$
\begin{gathered}
\mathcal{L}_{\mathrm{LV} \operatorname{dim} 6}^{\text {matter }}=\frac{1}{M^{2}} \int d^{4} \theta\left[\bar{\Phi}_{ \pm} e^{ \pm 2 e V} \Phi_{ \pm}\left(A_{ \pm}^{\mu \nu} D \sigma_{\mu \nu} W+\bar{A}_{ \pm}^{\mu \nu} \bar{D} \bar{\sigma}_{\mu \nu} \bar{W}\right)\right. \\
\left.+S_{ \pm}^{\mu \nu} \bar{\Phi}_{ \pm} e^{ \pm 2 e V}\left\{\nabla_{\mu}, \nabla_{\nu}\right\} \Phi_{ \pm}+Z^{\mu \nu} \Phi_{-}\left\{\nabla_{\mu}, \nabla_{\nu}\right\} \Phi_{+}+\bar{Z}^{\mu \nu} \bar{\Phi}_{-}\left\{\nabla_{\mu}, \nabla_{\nu}\right\} \bar{\Phi}_{+}\right],
\end{gathered}
$$

where $S_{ \pm}^{\mu \nu}$ are real symmetric traceless matrices, $Z^{\mu \nu}$ is a complex symmetric traceless matrix and $\bar{Z}^{\mu \nu}$ is its complex conjugate.

In this section, we do not give the explicit component expressions of these supersymmetric operators, but it is not hard to see that operators like $F_{\mu \rho} F_{\nu \sigma} F^{\rho \sigma}$ or $F_{\rho \sigma} F^{\rho \sigma} F_{\mu \nu}$, do not arise. This might seem surprising, since such terms do appear in investigations of non-commutative SUSY models, and SQED in particular [35, 36]. However, there is no inconsistency: as pointed out in [36] the Seiberg-Witten map for non-commutative supersymmetric gauge theories cannot simultaneously have local and chiral gauge transformations and be invariant under conventional supersymmetry. In our construction we have insisted on these three principles. Thus our framework is more restrictive and does not allow for the operators cubic in the electromagnetic field strength. 


\section{QUANTUM CORRECTIONS IN THE PRESENCE OF LV}

\section{A. Absence of a LV induced D-term}

In this subsection we want to show that the dimension five LV operators discussed in section \A do not lead to dangerous power law divergences in SQED. Before we enter this analysis, we would like to emphasize why this is an important issue.

One of the main reasons supersymmetry is conventionally introduced is that supersymmetric theories are free of destabilizing quadratic divergences. There is of course one wellknow exception to this assertion, the $D$-term of a $U(1)$ vector multiplet, which is in principle quadratically divergent at one loop. However, in any supersymmetric theory that is free of anomalies the coefficient in front of the $D$ term vanishes identically. The introduction of higher dimensional LV operators could upset the fine balance of the cancellation of the $D$ term, reintroducing a quadratic divergence. We will now show that such destabilizing effects do not arise.

To begin this investigation, we specify the relevant Feynman rules. The matter LV operators (92) can be decomposed into a modification of the kinetic term of the chiral multiplets

$$
\int d^{4} \theta \bar{\Phi}_{ \pm}\left(1+\frac{N_{ \pm}^{\mu}}{M} i \partial_{\mu}\right) \Phi_{ \pm}
$$

and their gauge interactions:

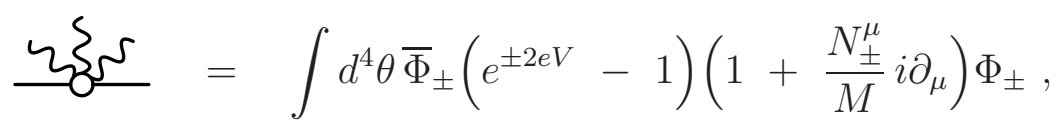

and

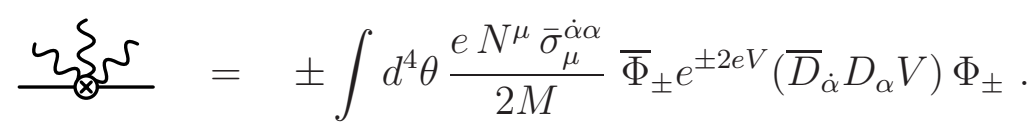

For most phenomenological applications it is sufficient to include only the first order terms

in expansion in LV parameters. For the study of the $D$-term, however, higher order terms in LV have to be taken into account as well. It proves useful to combine quadratic terms (19) into re-summed propagators

$$
\simeq=\frac{1}{\square} \frac{1}{1+\frac{N_{ \pm}^{\mu}}{M} i \partial_{\mu}} \text {. }
$$

These propagators are better behaved in the UV, because of the additional derivative in the denominator. Since the momentum scale involved in the $D$-term calculation is far above the 
soft breaking scale, we ignore soft scalar masses and the electron mass. The LV parts of the re-summed propagators are canceled exactly by the corresponding parts of the interactions (201), when these propagators are attached to their $\Phi_{ \pm}$-legs. Diagrammatically this may be represented as

$$
\text { 235 }
$$

This shows that for a single insertion of the interactions (20) and (21) only the latter survives, leading to logarithmic renormalization of the dimension five LV operators, which will be studied in the next subsection. Another immediate consequence of (23) is that LV does not modify the cancellation of the Fayet-Iliopoulos (FI) $D$-term at one loop. Indeed, the $\bar{D}_{\dot{\alpha}} D_{\alpha} V$ - proportional interaction gives a total derivative in the superspace when $\Phi_{ \pm}$fields are integrated out, and thus vanishes. The part of interaction (20) linear in $V$ could induce the $D$-term via the tadpole diagrams obtained by closing the chiral loop in the diagrams above. However, cancellation property (23) reduces the tadpole with LV to a standard tadpole diagram of the Lorentz-preserving case,

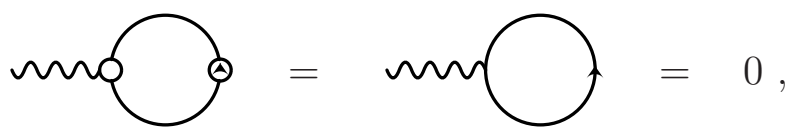

where the last diagram gives a vanishing $D$-term when both $\Phi_{+}$and $\Phi_{-}$loops are taken into account. More generically, the $D$-term will vanish for any chiral field content, provided that the sum of all charges of chiral fields is zero. Hence, to first order in the LV parameters, no extra quadratic divergences are introduced into SQED by LV interactions.

The situation becomes more complicated if we go to higher orders in the LV parameters and to higher loop orders: the arguments presented above are sufficient to prove that to all orders no quadratic divergences arise, as long as we ignore the second interaction structure (21). At two-loop level vertex (21) introduces additional factors of $\bar{D}_{\dot{\alpha}} D_{\alpha}$ into diagrams, and thereby raises the degree of divergence of a diagram by one, as $\left\{\bar{D}_{\dot{\alpha}}, D_{\alpha}\right\}=-2 i \sigma_{\alpha \dot{\alpha}}^{\mu} \partial_{\mu}$. Unlike in the one-loop calculation of the FI tadpole, the $\bar{D}_{\dot{\alpha}} D_{\alpha}$ derivatives may now act inside the diagrams, and hence still can lead to a potential power-like divergence. In addition, each of the internal propagator lines may be dressed with multiple LV insertions.

Even though the cancellation property we relied upon at one loop, Eq. (23)), does not apply here, luckily, one can show that at two (and higher) loops the effects of all such possible insertions still cancel. The proof of this statement is similar to the proof in a standard 
Lorentz preserving U(1) theory [39]. At two loops, there are two types of diagrams,

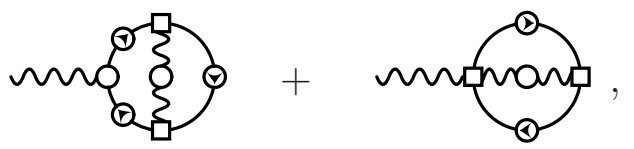

where the vertices with boxes denote either regular gauge interactions or the ones given in (21) with the derivatives $\bar{D}_{\dot{\alpha}} D_{\alpha}$ acting on the internal gauge lines. Using the diagrammatic result (23), the vertex of the first diagram with the external $V$ line and one adjacent chiral line can be turned into an ordinary Lorentz-preserving combination. By partial integration on the internal gauge line all (LV) operators can be moved away as far as possible from the vertex with the external gauge multiplet. After these manipulations the diagrams can be represented pictorially as

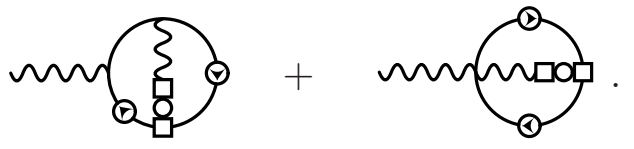

After some straightforward algebra involving $\bar{D}^{2}$ and $D^{2}$ along the chiral field propagators, one can show that the ordinary chiral line in the first diagram of (26) can be reduced to a delta function in the superspace. This makes both diagrams in (26) identical in structure, but with opposite signs. Thus, we observe that these diagrams indeed cancel, and no FI $D$-term arises even at two (or higher) loop level.

\section{B. Absence of LV induced gauge anomalies and of the Chern-Simons term}

It is well known that anomalies put severe restrictions on the matter spectrum of particle physics models. One may wonder whether LV might lead to new anomalies. Should this happen, either the LV vectors must be restricted by stringent conditions that ensure the anomaly cancellation, or gauge non-invariant terms would have to be included in the classical action in order to cancel the gauge variation of the effective action obtained by integrating out the fermions. In the SUSY LV context the supersymmetric extension of the CS term would be a possible term that could cancel new anomalies. We will show now that LV terms at dimension five do not modify the chiral anomaly. As a consequence, there are no further restrictions on the LV vectors and the local gauge non-invariant SUSY CS term (6) is not admissible. In addition to this indirect anomaly argument against the CS term, we show that it is not generated by explicitly computing relevant diagrams. 
To prove the claim that there are no new gauge anomalies, we closely follow the computation of the covariant anomaly presented in refs. [40, 41] using the techniques developed by Fujikawa and Konishi [42, 43]. We consider the classical LV chiral multiplet action

$$
S=\int d^{8} z \bar{\Phi}_{+} e^{2 e V}\left(1+i N_{+}^{\mu} \nabla_{\mu}\right) \Phi_{+} .
$$

The variation of the effective action, obtained by integrating out the chiral multiplet $\Phi_{+}$, under a chiral gauge transformation $(\delta \Lambda \neq 0, \delta \bar{\Lambda}=0)$, is given by

$$
\delta_{\Lambda} \Gamma(V)=\left\langle\delta_{\Lambda} S\right\rangle=2 e\left\langle\int d^{8} z \bar{\Phi}_{+} e^{2 e V}\left(1+i N_{+}^{\mu} \nabla_{\mu}\right)\left(\delta \Lambda \Phi_{+}\right)\right\rangle .
$$

This expression is regularized by inserting the operator $\exp \left(\square_{+} / M^{2}\right)$, where $\square_{+}$is the covariant d'Alembertian that preserves chirality, and $M$ is a regulator mass which will be taken $M \rightarrow \infty$ at the end of the computation. To evaluate the regularized amplitude we determine the propagator in the background field $V$ :

$$
\left\langle\Phi_{+2} \bar{\Phi}_{+1} e_{1}^{2 e V}\right\rangle=i\left(1+i N_{+}^{\mu} \frac{1}{16 \square_{+}} \bar{\nabla}^{2} \nabla^{2} \nabla_{\mu}\right)_{2}^{-1}\left(\frac{1}{16 \square_{+}} \bar{\nabla}^{2} \nabla^{2}\right)_{2} \delta_{21}^{8} .
$$

Here the subscripts 1 and 2 indicate that the corresponding expression is a function (or derivative) of the superspace coordinates $z_{1}$ and $z_{2}$. By inserting this propagator in the variation of the effective action (28), one can show that the LV factors exactly cancel out, and the anomaly reduces to the standard one without any LV. Notice that in this derivation we have not used any properties of the operator $i N_{+}^{\mu} \nabla_{\mu}$ except that it is gauge covariant. Therefore, this argument shows that no kinetic modification ever leads to new anomaly constraints.

Because the gauge anomaly is the same as in the Lorentz preserving theory (and therefore absent in LV SQED), we conclude that no CS term can be generated by quantum effects. The reason is that the local version of the SUSY CS (6) is not gauge invariant, see (17). The gauge invariant version of the SUSY CS (8) is, in its turn, nonlocal. Since only local and gaugeinvariant counterterms can arise in a non-anomalous quantum field theory, neither version of super-CS can get induced. This result can be confirmed by a direct loop computation: the diagrams of Fig. (1) can potentially generate the SUSY CS term ([6), but an explicit calculation reveals that all these contributions cancel for both $N_{+}^{\mu}$ and $N_{-}^{\mu}$ backgrounds, even when the electron mass $m_{e}$ is retained. The absence of CS term induced by quantum effects ([6) can be understood as a SUSY version of the no-go theorem of Coleman and Glashow 2]. In section IVB we show that the CS term is also not induced by the soft supersymmetry breaking. 


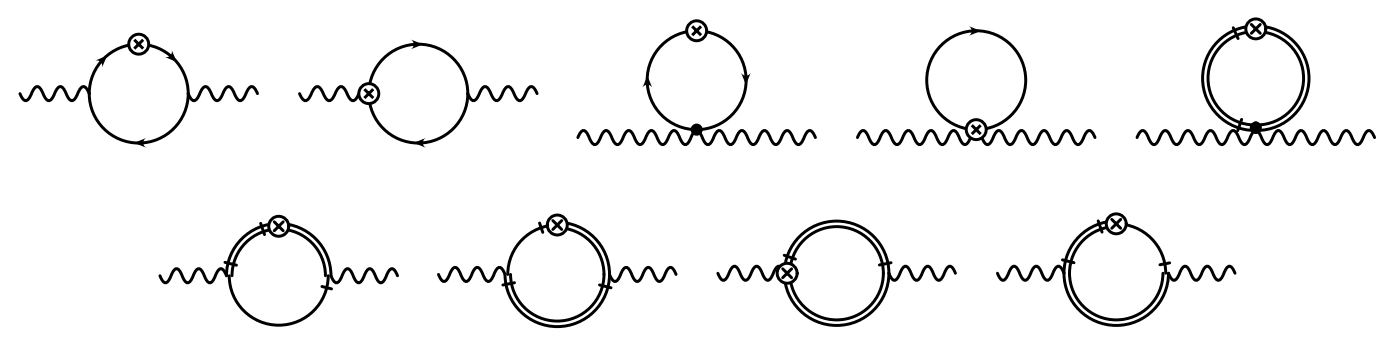

Figure 1: LV diagrams in massive SQED. Solid lines denote chiral field propagators, wiggled line represent external gauge superfield legs. Crossed circles indicate insertions of the LV operator (9). Double lines represent chirality-flipping propagators $\langle\Phi \Phi\rangle$ and $\langle\bar{\Phi} \bar{\Phi}\rangle$. Bars denote the $\bar{\Phi}$ ends of the propagators. Only the $N_{+}^{\mu}$ operator is included in this figure; the $N_{-}^{\mu}$ operator generates the same set of diagrams.

\section{RGE evolution of dimension five LV operators in SQED}

As mentioned in the Introduction, within an effective field theory approach, we are allowed to assume that operators (91), (11) and (13) are generated at the UV scale $M$ by some unspecified LV dynamics. All experimental limits are obtained at much lower energy scales. Therefore, in order to derive meaningful experimental constraints on parameters of LV SQED, we have to evolve the LV operators down to the low-energy scale. Furthermore, we know that SUSY is broken, and the operators of dimension five will source dimension three LV operators via SUSY breaking, leading to tight bounds on LV parameters of the model. In this section, we derive and solve the renormalization group equations (RGE's) for dimension five LV operators assuming unbroken SUSY. In the next section we include the effects of soft breaking and calculate resulting dimension three operators.

We work in the linear approximation in LV parameters, and neglect all terms that involve higher powers of $1 / M$. The running of the LV operators (9), (11) and (13) is, in part, a consequence of the wave function renormalization of various superfields induced by standard SQED one-loop diagrams. We do not give them explicitly here, but we take their effects into account in the resulting RGE's. For the logarithmic running of the LV parameters above the supersymmetric threshold, we can ignore soft breaking masses and electron mass inside loops. At one loop, this means that loop diagrams with internal lines of $\Phi_{+}$and $\Phi_{-}$can be calculated independently.

The renormalization of the electron/positron LV operators (9) is induced by the diagrams 


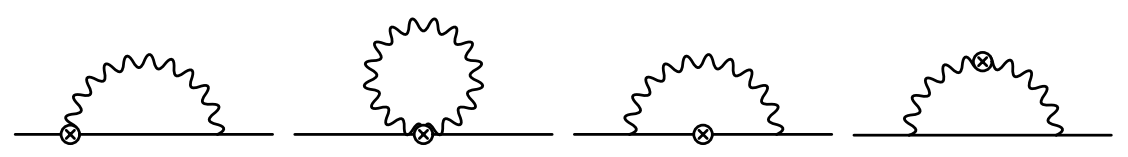

Figure 2: One-loop corrections to the matter multiplet operators (91). The notations are the same as in Fig. 1 Wiggled lines represent the gauge superfield propagators, and the crossed circles are insertions of the LV operators (9) or (11).

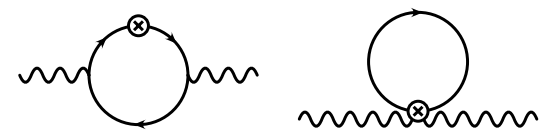

Figure 3: One-loop corrections to the gauge LV operator (11) using the same pictorial notation as in Fig. 2

shown in Fig. 2. The first two diagrams involve the interactions (21). Notice that the seagull diagram vanishes because the photon superfield loop contains only two super covariant derivatives. The last diagram is induced by the operator (11). Loops with a single insertion of tensor interaction (13) vanish identically, as there are no operators in the chiral sector that can couple to $T^{\mu \nu \lambda}$.

In the gauge sector we find that the renormalization of the tensor LV gauge operator (13) is absent. Indeed, since we work in the first order in LV, this operator cannot receive any corrections from operators that depend on vector backgrounds. The renormalization of LV gauge operator, with $N^{\mu}$ (11), is given by the diagrams shown in Fig. 3. Again, one can use the cancellation property (23) to observe that the vertex in the second diagram of Fig. 3 is given by (21) only. The combination of these gauge self-energy diagrams is only logarithmically divergent, which gives another reason why the dimension three LV CS term is not generated by loop effects in this approximation.

After a straightforward calculation of logarithmically divergent parts of the diagrams in Figs. 2] and 3, and inclusion of wave function renormalization effects, we arrive at the 
renormalization group equation (RGE) for the LV parameters:

$$
\mu \frac{\partial}{\partial \mu}\left(\begin{array}{c}
N^{\nu} \\
N_{+}^{\nu} \\
N_{-}^{\nu} \\
T^{\mu \nu \rho}
\end{array}\right)=\frac{\alpha}{2 \pi}\left(\begin{array}{rrrr}
2 & -1 & -1 & 0 \\
-6 & 3 & 0 & 0 \\
-6 & 0 & 3 & 0 \\
0 & 0 & 0 & 2
\end{array}\right)\left(\begin{array}{c}
N^{\nu} \\
N_{+}^{\nu} \\
N_{-}^{\nu} \\
T^{\mu \nu \rho}
\end{array}\right),
$$

As usual, $\alpha=e^{2} /(4 \pi)$ denotes the fine structure constant. The $(1,1)$ and $(4,4)$ elements of the matrix in (30) are equal and result only from the renormalization of wave functions. The electron and positron LV parameters $N_{ \pm}^{\mu}$ both give and receive equal contributions to and from the vector LV parameter $N^{\mu}$. This explains why the pairs of matrix elements $(1,2)$ and $(1,3),(2,2)$ and $(3,3)$, and $(2,1)$ and $(3,1)$ are equal.

It will prove useful to introduce the following combinations of LV parameters that couple to operators of definite parity:

$$
N_{V}^{\mu}=\frac{N_{+}^{\mu}-N_{-}^{\mu}}{2}, \quad N_{A}^{\mu}=\frac{N_{+}^{\mu}+N_{-}^{\mu}}{2} .
$$

$N_{V}^{\mu}$ and $N_{A}^{\mu}$ are the charge conjugation odd and even combinations, respectively. In general, vector backgrounds do not need to have the same orientation in Minkowski space, and the off-diagonal elements of the renormalization group coefficients in (30) mix them, resulting in changes of their directions. By diagonalizing (30) we identify a set of eigenvectors

$$
N_{1}^{\mu}=N_{V}^{\mu}, \quad N_{2}^{\mu}=3 N^{\mu}-2 N_{A}^{\mu}, \quad N_{3}^{\mu}=2 N^{\mu}+N_{A}^{\mu},
$$

that evolve under the RGE independently; each of them may change its size but not its direction. $N_{V}^{\mu}$ renormalizes independently because it is the only combination that is odd under the charge conjugation. In this basis, the RGE's and their solutions are given by

$$
\mu \frac{\partial}{\partial \mu} N_{i}^{\nu}=\lambda_{i} \frac{\alpha}{2 \pi} N_{i}^{\nu} \quad \Rightarrow \quad N_{i}^{\nu}(\mu)=\left(\frac{\alpha(\mu)}{\alpha(M)}\right)^{\frac{\lambda_{i}}{2}} N_{i}^{\nu}(M),
$$

where the eigenvalues read $\lambda_{i}=\left(\lambda_{1}, \lambda_{2}, \lambda_{3}\right)=(3,6,-1)$. To obtain these solutions we have used the standard SQED beta function $\mu \frac{\partial}{\partial \mu} \alpha=\frac{1}{\pi} \alpha^{2}$.

Within the SQED framework, the renormalization effects of these LV parameters are small: even if we take $\mu=m_{s} \approx 1 \mathrm{TeV}$ and $M=M_{\mathrm{Pl}} \approx 10^{19} \mathrm{GeV}$, the running affects the $\mathrm{LV}$ parameters by only about $10 \%$. In other words, the linearized version of (133)

$$
N_{i}^{\nu}\left(m_{s}\right) \simeq\left(1-\frac{\lambda_{i} \alpha}{2 \pi} \log \left(M / m_{s}\right)\right) N_{i}^{\nu}(M)
$$


gives a good approximation of the exact answer. The same conclusion holds for the running of the irreducible tensor $T^{\lambda \mu \nu}$. Although it may look as though a 10 percent level change in $N_{i}^{\mu}$ is insignificant, one should keep in mind, that in a more realistic framework of MSSM the number of charged degrees of freedom running inside the loops is significantly larger than in SQED, which would lead to appreciable changes in LV parameters between the Planck and the weak scales. Nevertheless, the main numerical change in the actual size of observable LV effects will result from soft SUSY breaking, as will be discussed in the next section.

\section{INDUCED DIMENSION THREE OPERATORS BY SOFT SUSY BREAKING}

Once SUSY is broken, dimension three LV operators can be induced with coefficients controlled by the soft-breaking mass scale. Following the usual approach (see e.g. Ref. [29]), we introduce spurion superfields $\left(\theta^{2}, \bar{\theta}^{2}\right)$ in superspace expressions. We consider only soft SUSY breaking in the matter sector. We ignore other soft-breaking terms, including a gaugino mass, which can be motivated by the most common MSSM scenarios. Generically, we can assume that parity is broken, so that the scalar partners of left- and right-handed electrons have different masses.

The possible soft SUSY breaking masses of the electron and positron can be written as

$$
\mathcal{L}_{S B}=-\int d^{2} \theta \theta^{2}\left(m_{s}^{0}\right)^{2} \Phi_{+} \Phi_{-}+\text {h.c. }-\int d^{4} \theta \theta^{2} \bar{\theta}^{2}\left(\left(m_{s}^{+}\right)^{2} \bar{\Phi}_{+} \Phi_{+}+\left(m_{s}^{-}\right)^{2} \bar{\Phi}_{-} \Phi_{-}\right),
$$

where $m_{s}^{ \pm}, m_{s}^{0}$ are real and complex masses, respectively. To make parity violation manifest in the SUSY breaking (35) we introduce

$$
\Delta m^{2}=\left(m_{s}^{+}\right)^{2}-\left(m_{s}^{-}\right)^{2}, \quad\left(m_{s}^{ \pm}\right)^{2}=m_{s}^{2} \pm \frac{\Delta m^{2}}{2} .
$$

The parity conserving scenario is obtained in the limit $\Delta m^{2} \rightarrow 0$. Throughout the paper we assume that $\Delta m^{2}$ is somewhat smaller but not necessarily much smaller than $m_{s}^{2}$, and that the values of the soft-breaking parameters are such that scalar electrons do not develop vacuum expectation values. Viewing SQED as a subset of MSSM, we can also neglect $\left(m_{s}^{0}\right)^{2}$, $\left(m_{s}^{0}\right)^{2} \sim O\left(m_{s} m_{e}\right) \ll m_{s}^{2}$.

Once SUSY is broken via (35) in the Lorentz-conserving sector, it will be communicated to the LV sector via loop corrections or on the equations of motion (EOM's), resulting in LV operators of dimension three. 
We start by listing all such operators in components, essentially extending the existing QED parametrization [1] to the SQED field content. In the matter sector these operators are

$$
\begin{aligned}
\mathcal{L}_{\mathrm{SB} \mathrm{LV} \mathrm{dim} 3}^{\text {matter }}= & 2 i \widetilde{A}_{+}^{\mu} \bar{z}_{+} \mathcal{D}_{\mu} z_{+}+2 i \widetilde{A}_{-}^{\mu} \bar{z}_{-} \mathcal{D}_{\mu} z_{-}+i \widetilde{C}^{\mu} z_{-} \mathcal{D}_{\mu} z_{+} \\
& +\widetilde{B}_{+}^{\mu} \bar{\psi}_{+} \bar{\sigma}_{\mu} \psi_{+}+\widetilde{B}_{-}^{\mu} \bar{\psi}_{-} \bar{\sigma}_{\mu} \psi_{-}+\widetilde{D}^{\mu \nu} \psi_{-} \sigma_{\mu \nu} \psi_{+} .
\end{aligned}
$$

In superfield notation they can be expressed as:

$$
\begin{gathered}
\mathcal{L}_{\mathrm{LV} \operatorname{dim} 3}^{\text {matter SB }}=\int d^{4} \theta \theta^{2} \bar{\theta}^{2}\left[2 i \widetilde{A}_{+}^{\mu} \bar{\Phi}_{+} \nabla_{\mu} \Phi_{+}-2 i \widetilde{A}_{-}^{\mu} \Phi_{-} \nabla_{\mu} \bar{\Phi}_{-}+\frac{1}{2}\left(\widetilde{C}^{\mu} \Phi_{-} \nabla_{\mu} \Phi_{+}+\text {h.c. }\right)\right. \\
\left.+\frac{1}{2} \widetilde{B}_{+}^{\mu} \bar{\nabla}_{+} \bar{\Phi}_{+} \bar{\sigma}_{\mu} \nabla \Phi_{+}+\frac{1}{2} \widetilde{B}_{+}^{\mu} \bar{\nabla} \bar{\Phi}_{-} \bar{\sigma}_{\mu} \nabla \Phi_{-}+\frac{1}{2}\left(\widetilde{D}^{\mu \nu} \nabla \Phi_{-} \sigma_{\mu \nu} \nabla \Phi_{+}+\text {h.c. }\right)\right] .
\end{gathered}
$$

The superfield expressions (38) for the operators (37) are not unique. One can use alternative spurion insertions inside gauge-invariant supersymmetric LV operators [23]. However, at the component level these expressions will reduce to linear combinations of the operators given in (38).

In the gauge sector there are only two LV operators of dimension three:

$$
\mathcal{L}_{\mathrm{SB} L V \operatorname{dim} 3}^{\text {gauge }}=\widetilde{E}_{\mu} \epsilon^{\mu \nu \rho \sigma} A_{\nu} \partial_{\rho} A_{\sigma}+\widetilde{F}_{\mu} \lambda \sigma^{\mu} \bar{\lambda}
$$

which can be rewritten in a superfield form using the CS superfield [44]:

$$
\mathcal{L}_{\mathrm{LV} \mathrm{dim} 3}^{\text {gauge }}=\int d^{4} \theta\left(\left(\widetilde{F}_{\mu}-\widetilde{E}_{\mu}\right) \theta^{4} W \sigma^{\mu} \bar{W}+\widetilde{E}_{\mu} \theta \sigma^{\mu} \bar{\theta}\left\{D^{\alpha}\left(V W_{\alpha}\right)+\bar{D}_{\dot{\alpha}} V \bar{W}^{\dot{\alpha}}\right\}\right)
$$

\section{A. Operators in the matter sector}

We now turn to the discussion of possible mechanisms that transmute dimension five SUSY LV operators into dimension three LV operators. There are two generic ways this may occur, at tree level via reduction over the EOM and via loop effects,

$$
\begin{aligned}
& {[L V]_{\operatorname{dim} 5} \stackrel{\text { EOM }}{\longrightarrow}\left(m_{s}^{2}+m_{e}^{2}\right)[L V]_{\operatorname{dim} 3}, \text { for selectrons }} \\
& {[L V]_{\operatorname{dim~} 5} \stackrel{1 \text { loop }}{\longrightarrow} \quad m_{s}^{2}[L V]_{\operatorname{dim} 3}, \text { for fermions and vector bosons . }}
\end{aligned}
$$

The tensor operator (13) does not mix with dimension three operators in any order in SUSY breaking, because there is simply no dimension three operator that can couple to $T^{\mu \nu \lambda}$. 


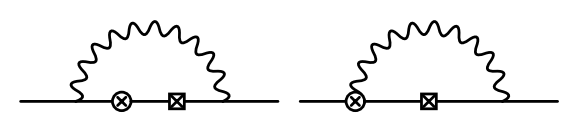

a

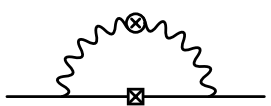

$\mathrm{b}$

Figure 4: Diagrams generating dimension three LV operators for electrons and positrons due to soft supersymmetry breaking and dimension five SUSY LV operators in gauge and matter sectors. In Figs. 4a and 40 the inserted operators are (9) and (11), respectively. Finally, the box with a cross denotes the insertion of the SUSY breaking operator (35).

Below we discuss in detail how dimension three operators are generated by tree level and loop effects.

The soft supersymmetry breaking (35) affects LV interactions for left- and right-handed selectrons already at tree level. The masses of scalar particles are lifted with respect to the masses of the electron and positron. This alters sfermions' equations of motion, leading to an enhancement of certain dimension three operators. Ignoring $\Delta m^{2}$ for a moment, one can easily show that the combination of LV operators (9) and SUSY breaking (35) leads to the following dimension three LV operator

$$
\mathcal{L}_{\text {sparticle }}^{\mathrm{EOM}}=\frac{N_{V}^{\mu}}{M} 2 i\left(m_{e}^{2}+m_{s}^{2}\right)\left\{\bar{z}_{+} \mathcal{D}_{\mu} z_{+}-\bar{z}_{-} \mathcal{D}_{\mu} z_{-}\right\}
$$

effectively generating the $\widetilde{A}_{ \pm}^{\mu}$-terms (37),

$$
\widetilde{A}_{ \pm}^{\mu}= \pm 2 \frac{N_{V}^{\mu}}{M}\left(m_{e}^{2}+m_{s}^{2}\right)
$$

However, we will not be interested in these particular operators due to the current impossibility to experimentally study the superpartner sector. In the matter sector only those operators involving electrons and positrons are important for phenomenology. For the same reason, we ignore the possible appearance of the operators proportional to $\widetilde{C}^{\mu}$, and in the gauge sector we will only be interested in the CS term that might be induced for photons.

At one loop level, the transmission of SUSY breaking to the LV sector of chiral fermions and gauge bosons may indeed be possible. We start with one loop effects in the matter sector. It is sufficient for our purposes to consider the running of dimension three operators within the interval of momenta $m_{s} \ll\left|p_{\text {loop }}\right| \ll M$, and to retain only the contributions enhanced by a large $\log \left(M / m_{s}\right)$, neglecting possible threshold corrections. To this accuracy, the soft breaking parameters inside loops can be treated as perturbations and inserted on 
internal lines of diagrams from Fig. 2, In Figs. 4a and $4 \mathrm{~b}$ we have inserted the dimension five SUSY LV operators (91) and (11), respectively.

Our one loop RGE analysis concentrates on the induced dimension three operators $\widetilde{B}_{\mu}$. Besides the contributions from diagrams in Figs. 4a and $4 \mathrm{~b}$, the complete set of RGE includes one loop running of the operator $\widetilde{B}_{\mu}$ itself, and its mixing with $\widetilde{A}_{\mu}$. The relevant set of RGE's includes:

$$
\begin{aligned}
\mu \frac{d \widetilde{A}_{+}^{\nu}}{d \mu} & =\frac{\alpha}{\pi}\left(\widetilde{A}_{+}^{\nu}-\widetilde{B}_{+}^{\nu}\right) \\
\mu \frac{d \widetilde{B}_{+}^{\nu}}{d \mu} & =\frac{\alpha}{2 \pi}\left(\widetilde{B}_{+}^{\nu}-\widetilde{A}_{+}^{\nu}+3 \frac{\left(m_{s}^{+}\right)^{2}}{M} N^{\nu}-2 \frac{\left(m_{s}^{+}\right)^{2}}{M} N_{+}^{\nu}\right) .
\end{aligned}
$$

Here we quote only the results for $\Phi_{+}$components; the extension to $\Phi_{-}$follows upon some simple substitutions. The requirement of exact SUSY at UV scale $M$ translates into the RGE boundary conditions: $\left.\widetilde{A}^{\mu}\right|_{M}=\left.\widetilde{B}^{\mu}\right|_{M}=0$. In addition, the full set of equations include the RGE's for the soft breaking masses,

$$
\mu \frac{d m_{s}^{2}}{d \mu}=\frac{\alpha}{4 \pi} m_{s}^{2},
$$

and RGE's for the dimension five SUSY LV operators (30).

Exact solutions of these RGE's are not warranted for our purposes. Instead, we use the same approximation as in (34) together with $\alpha / \pi \log \left(M / m_{s}\right)<1$, to obtain the solution

$$
\widetilde{B}^{ \pm \nu}\left(m_{s}\right)=\frac{\alpha}{\pi} \log \left(M / m_{s}\right) \frac{\left(m_{s}^{ \pm}\right)^{2}}{M}\left\{\frac{3}{2} N^{\nu}(M)-N_{ \pm}^{\nu}(M)\right\}
$$

for $\widetilde{B}_{\mu}^{ \pm}$in the leading $\alpha \log$ approximation.

\section{B. Operators in the gauge sector. Chern-Simons term.}

The absence of optical activity effects caused by the CS term has been checked over cosmological distances, providing a very sensitive probe of $k_{\mu}$ in (11) (see e.g. Ref. [10] and references therein). The limit on $k_{\mu}$ is about the present Hubble expansion rate, and is ten orders of magnitude better than the level of sensitivity for the best terrestrial experiments searching for LV parameters in (11). Not surprisingly, the issue of CS term generated by radiative corrections from other LV interactions has drawn a lot of interest [2, 45, 46, 47,, 48], exhibiting the whole range of answers for $k_{\mu}$ (including zero) being induced by $b_{\mu}$. A no-go 

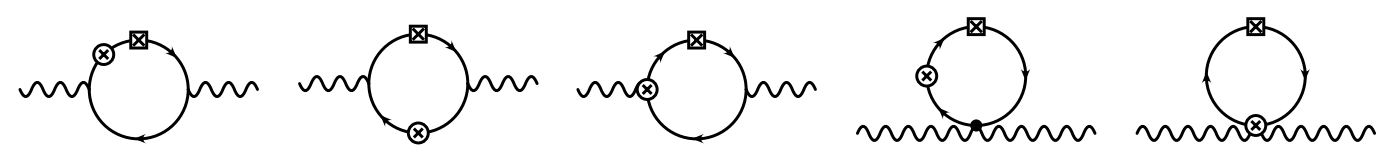

Figure 5: Dimension three one-loop contributions arising from the dimension five LV operators (9) and soft supersymmetry breaking.

theorem by Coleman and Glashow 2] indicates the absence of the radiatively generated CS term. If suitably rephrased, it states that the CS term cannot be induced to first order by gauge invariant LV interactions. In section $\amalg$ w we have extended this theorem to the exact SUSY LV interactions.

We would like to argue that below the soft SUSY breaking scale the CS term also cannot be generated. Indeed, the CS interaction can only be generated by a fermion running in the loop, as a bosonic loop cannot produce $\epsilon^{\mu \nu \rho \sigma}$ entering the expression for the CS term. However, the SUSY breaking terms (35) only provide masses for the bosonic components of chiral superfields and thus only affect the scalar parts of the diagrams, which are incapable of inducing the CS interaction. (The possibility of a soft gaugino mass is not relevant because diagrams that could induce the CS term only include chiral matter fermions, not gauginos.) In particular, the no-go theorem by Coleman and Glashow [2] for QED is re-obtained by sending the soft masses to infinity.

We have confirmed this result by a direct calculation in the presence of soft-breaking. The relevant diagrams, given in Fig. 5, are obtained by inserting the soft breaking interaction (35) into the diagrams shown in Fig. 3. As for the direct confirmation of the absence of the SUSY CS term in section [IIB instead of calculating all possible terms, we have only concentrated on those structures that can induce the CS term. Here, again, the vertex cancellation property (23) can be used quite effectively to mutually cancel contributions of particular diagrams. A straightforward calculation shows that all terms proportional to the CS interaction indeed cancel.

Note that this statement is only valid for the pure CS term $\epsilon^{\mu \nu \rho \sigma} A_{\nu} \partial_{\rho} A_{\sigma}$, while there is no evidence against the other possible operator in the photon sector, $\lambda \sigma^{\mu} \bar{\lambda}$. However, the presence/absence of the latter term is obviously not very relevant for phenomenological applications. 


\section{PHENOMENOLOGY OF LV SQED: LV OBSERVABLES AND EXPERIMENTAL LIMITS}

\section{A. Component Expressions for LV operators}

In order to derive phenomenological consequences of the LV operators, we need to obtain their component expressions. First we consider the matter operators (9). The component form for the electron part is given by:

$$
\begin{gathered}
\mathcal{L}_{\mathrm{LV} \operatorname{dim} 5}^{\text {matter }(+)}=\frac{N_{+}^{\mu}}{M}\left[i \bar{F}_{+} \mathcal{D}_{\mu} F_{+}+i e \bar{z}_{+} D \mathcal{D}_{\mu} z_{+}-i e \mathcal{D}_{\mu}\left(\bar{z}_{+}\right) D z_{+}+\frac{1}{2} \bar{\psi}_{+} \mathcal{D}_{(\mu} \mathcal{D}_{\nu)} \bar{\sigma}^{\nu} \psi_{+}\right. \\
+i e \frac{\sqrt{2}}{2}\left\{\bar{\psi}_{+} \bar{\sigma}_{\mu} \lambda F_{+}-\bar{F}_{+} \bar{\lambda} \bar{\sigma}_{\mu} \psi_{+}\right\}+e^{2} \bar{z}_{+}\left\{\lambda \sigma_{\mu} \bar{\lambda}-\bar{\lambda} \bar{\sigma}_{\mu} \lambda\right\} z_{+}+\frac{1}{2} e \bar{\psi}_{+} \bar{\sigma}_{\mu} D \psi_{+} \\
-\sqrt{2} e\left\{\mathcal{D}_{\mu}\left(\bar{\psi}_{+}\right) \bar{\lambda} z_{+}+\bar{z}_{+} \lambda \mathcal{D}_{\mu} \psi_{+}\right\}-\frac{\sqrt{2}}{2} e\left\{\bar{\psi}_{+} \bar{\sigma}^{\nu} \sigma_{\mu} \bar{\lambda} \mathcal{D}_{\nu} z_{+}+\mathcal{D}_{\nu}\left(\bar{z}_{+}\right) \lambda \sigma_{\mu} \bar{\sigma}^{\nu} \psi_{+}\right\} \\
\left.-\frac{1}{4} e \bar{\psi}_{+} \epsilon_{\mu}{ }^{\nu \rho \sigma} F_{\rho \sigma} \bar{\sigma}_{\nu} \psi_{+}+i \bar{z}_{+} \mathcal{D}^{\nu} \mathcal{D}_{\mu} \mathcal{D}_{\nu} z_{+}+\frac{1}{2} i e \mathcal{D}_{\nu}\left(\bar{z}_{+}\right) \epsilon_{\mu}{ }^{\nu \rho \sigma} F_{\rho \sigma} z_{+}\right],
\end{gathered}
$$

This component representation of dimension $5 \mathrm{LV}$ operators allows for further reduction of several terms in (46) on the equations of motion. The result for this tedious but routine reduction is given in Appendix A. To facilitate phenomenological applications, we convert all Weyl spinors into Dirac/Majorana four component spinors:

$$
\Psi=\left(\begin{array}{c}
c \psi_{+} \\
\psi_{-}
\end{array}\right), \quad \text { and } \quad \lambda=\left(\begin{array}{c}
c \lambda \\
\bar{\lambda}
\end{array}\right) \text {. }
$$

Using notations (31) and (36), we present the dimension five LV operators (9), containing electron and photon fields, as:

$$
\mathcal{L}_{\mathrm{LV}}^{\text {matter }}=-\frac{N_{A}^{\mu}}{M} \frac{1}{2} e \bar{\Psi} \widetilde{F}_{\mu \nu} \gamma_{\nu} \Psi-\frac{N_{V}^{\mu}}{M} \frac{1}{2} e \bar{\Psi} \widetilde{F}_{\mu \nu} \gamma^{\nu} \gamma^{5} \Psi+\frac{N_{V}^{\mu}}{M} m_{e}^{2} \bar{\Psi} \gamma_{\mu} \Psi
$$

Using the same notation, the dimension three operators (45) can be rewritten as a vector and axial-vector operators:

$$
\begin{aligned}
\mathcal{L}_{\mathrm{LV} \operatorname{dim} 3}^{\text {matter }}= & -\bar{\Psi} \gamma^{\mu} \Psi\left\{m_{s}^{2} N_{V}^{\mu}+\frac{\Delta m^{2}}{2} N_{A}^{\mu}-\frac{3}{2} \frac{\Delta m^{2}}{2} N^{\mu}\right\} \frac{\alpha \log \left(M / m_{s}\right)}{\pi M} \\
& -\bar{\Psi} \gamma^{\mu} \gamma^{5} \Psi\left\{m_{s}^{2} N_{A}^{\mu}+\frac{\Delta m^{2}}{2} N_{V}^{\mu}-\frac{3}{2} m_{s}^{2} N^{\mu}\right\} \frac{\alpha \log \left(M / m_{s}\right)}{\pi M} .
\end{aligned}
$$

The next operator to consider in components is the photon operator (11):

$$
\mathcal{L}_{\mathrm{LV} \operatorname{dim} 5}^{\text {gauge }(\mathrm{K})}=\frac{N^{\mu}}{M}\left(2 \bar{\lambda} \gamma_{\mu} \square \lambda+2 \lambda \partial_{\mu} \not \partial \bar{\lambda}-2 D \partial^{\nu} F_{\mu \nu}+\partial_{\lambda} F^{\lambda \nu} \widetilde{F}_{\nu \mu}\right) .
$$


These components are reducible on the equations of motion, and only the last term in (50) leads to a contribution in the electron and photon sectors. By substituting $\partial_{\lambda} F^{\lambda \mu}$ with the electromagnetic current $J_{E M}^{\mu}=-e \bar{\Psi} \gamma^{\mu} \Psi$, we get an interaction term

$$
\mathcal{L}_{\text {gauge }(\mathrm{K})}^{\mathrm{EOM}}=e \bar{\Psi} N^{\mu} \gamma^{\nu} \widetilde{F}_{\mu \nu} \Psi
$$

which has the same form as the second term in (48). Notice, however, that this coincidence holds only within QED, as in the full MSSM $J_{E M}^{\mu}$ will also have other (i.e. hadronic) contributions. Finally, the tensor operator (13) has the following component expression

$$
\mathcal{L}_{\mathrm{LV} \operatorname{dim} 5}^{\text {gauge }(\mathrm{T})}=\frac{2 T_{\mu \nu \rho}}{M}\left(F^{\nu \lambda} \partial^{\mu} F_{\lambda}^{\rho}-D \partial^{\mu} \widetilde{F}^{\nu \rho}-\bar{\lambda} \partial^{\mu} \partial_{\sigma} \bar{\sigma}_{\tau} \lambda \epsilon^{\sigma \tau \nu \rho}\right) .
$$

It can be reduced on the equations of motion by using integration by part and Jacobi identities. Applying the equations of motion to the pure electromagnetic field strength term in (52), we obtain in the electron-photon sector of QED

$$
\mathcal{L}_{\text {gauge (T) }}^{\mathrm{EOM}}=2 e T_{\mu \nu \rho} \bar{\Psi} \gamma^{\mu} F^{\nu \rho} \Psi
$$

Confirming the general conclusion of [23], we observe that none of the LV operators give corrections to the EOM that grow at high energies.

Now we gather all operators of phenomenological interest of dimensions five and three, (49), (48), (51) and (53), in a single expression:

$$
-\mathcal{L}_{\mathrm{eff} L V}=\bar{\Psi} \gamma^{\mu}\left(a_{\mu}+b_{\mu} \gamma^{5}+e c_{\nu} \widetilde{F}^{\nu \mu}+e d_{\nu} \widetilde{F}^{\nu \mu} \gamma^{5}+e f_{\mu \rho \sigma} F^{\rho \sigma}\right) \Psi
$$

where we use the notations of [49] for the coefficients of dimension three operators. The Wilson coefficients in (54) are expressed in terms of the original LV parameters, electromagnetic coupling constant, and soft breaking masses:

$$
\begin{aligned}
a^{\mu} & =-\frac{1}{M} m_{e}^{2} N_{V}^{\mu}+\frac{\alpha \log \left(M / m_{s}\right)}{\pi M}\left\{m_{s}^{2} N_{V}^{\mu}+\frac{\Delta m^{2}}{2} N_{A}^{\mu}-\frac{3}{2} \frac{\Delta m^{2}}{2} N^{\mu}\right\}, \\
b^{\mu} & =\frac{\alpha \log \left(M / m_{s}\right)}{\pi M}\left\{m_{s}^{2} N_{A}^{\mu}+\frac{\Delta m^{2}}{2} N_{V}^{\mu}-\frac{3}{2} m_{s}^{2} N^{\mu}\right\}, \\
c^{\mu} & =\frac{1}{M}\left\{\frac{1}{2} N_{A}^{\mu}-N^{\mu}\right\}, \quad d^{\mu}=\frac{1}{M} \frac{N_{V}^{\mu}}{2}, \quad f^{\mu \nu \rho}=\frac{2}{M} T^{\mu \nu \rho} .
\end{aligned}
$$

The result is given in the leading $\alpha$ log approximation, and with all LV parameters normalized at the SUSY threshold $m_{s}$. The operator proportional to $a^{\mu}$ does not lead to any physical effects as it can be totally absorbed into the kinetic term $-i \bar{\Psi} \not \partial \Psi$ via a phase redefinition, $\Psi(x) \rightarrow e^{i a^{\mu} x_{\mu}} \Psi(x)$. The rest of the operators lead to observable LV signatures. 


\section{B. Constraints on LV parameters of SQED}

Now, we are prepared to extract observational consequences of LV SQED, and to impose constraints on the coefficients of the effective low-energy Lagrangian (154). To do that, we derive the non-relativistic effective Hamiltonian corresponding to (54) by splitting the external backgrounds into spatial and time-like components:

$$
\begin{aligned}
\mathcal{H}_{\mathrm{eff}}= & \frac{\vec{p} \cdot \vec{a}}{m}+\vec{b} \cdot \vec{\sigma}+\left\{\frac{e \vec{p}}{m},[\vec{c} \times \vec{E}]-c^{0} \vec{B}\right\}-e d^{0}(\vec{B} \cdot \vec{\sigma}) \\
& +e \vec{d} \cdot[\vec{E} \times \vec{\sigma}]+\left\{\frac{e p^{k}}{m}, 2 f_{k 0 l} E^{l}+f_{k l m} \epsilon_{l m n} B^{n}\right\} .
\end{aligned}
$$

Here $\vec{p}=-i \hbar \vec{\partial}$ is the momentum operator and $\{.,$.$\} denotes the anti-commutator.$

The tightest constraints come from the experiments searching for abnormal spin precession around external directions determined by the LV vectors (55). These experiments limit LV parameters for electrons and nucleons. The relevant parameter we should compare our estimates with is the energy shift due to LV effects $\Delta \omega_{L V}$. In LV SQED, the effects are mediated by dimension five operators, and therefore the strength of the constraints on combinations of $M$ and LV backgrounds depends very sensitively on the energy scale $\mu$ relating $\Delta \omega_{L V}$ and $M^{-1}, \Delta \omega_{L V} \sim \mu^{2} M^{-1}$. Our analysis shows that several possibilities for $\mu$ are possible: the soft breaking scale, the hadronic scale (i.e. $\Lambda_{Q C D}$ ), electron mass, and finally, the energy scale given by an external magnetic or electric field.

\section{$L V$ electron spin precession}

The soft breaking scale enters in the LV parameter $b_{\mu}$, which is limited by torsion balance experiments searching for LV in the electron sector [50]. The sensitivity to the spatial part of the axial-vector coupling $b^{i}$ achieved in this experiments is at the level better than $\left|b^{i}\right|<10^{-28} \mathrm{GeV}$. This condition imposes a stringent constraint on the combination of the soft breaking masses, $M$, and LV parameters:

$$
\frac{m_{s}^{2}}{(100 \mathrm{GeV})^{2}} \frac{10^{19} \mathrm{GeV}}{M}\left|N_{A}^{i}-\frac{3}{2} N^{i}+\delta_{s} N_{V}^{i}\right|<10^{-12},
$$

where we normalize $M$ to the Planck scale. We have introduced a dimensionless quantity $\delta_{s}=\Delta m^{2} /\left(2 m_{s}^{2}\right)$ that parameterizes parity violation in the soft breaking sector. The lightest values for $m_{s}$ not excluded by direct collider searches are slightly above $100 \mathrm{GeV}$, and therefore $\left|N_{A}^{i}-\frac{3}{2} N^{i}\right|$ is limited to be less than $10^{-12}$. 


\section{LV nuclear spin precession}

The next constraint uses the energy scale $\mu \sim \Lambda_{Q C D}$ from hadron physics. In order to obtain it, we have to go beyond pure QED and include hadronic components in $J_{E M}^{\mu}$ (51). Then, as discussed earlier in [23], the LV SQED operator (111) gives rise to interaction of the spatial components of $N^{\mu}$, an electric field, and the spatial component of the hadronic current, $\mathcal{L}=M^{-1} \epsilon_{i j k} N^{i} E^{j} J_{E M}^{k}$. The average of such interaction inside the nucleus with spin $I$ leads to the effective Hamiltonian $\mathcal{H}_{\mathrm{eff}}=\kappa \vec{I} \cdot \vec{N}$. The strength $\kappa$ of this interaction is given by a nuclear matrix element, which can be estimated as the product of the typical value of the electric field inside a heavy nucleus times the characteristic nucleon momentum. Combined with a $10^{-32} \mathrm{GeV}$ level of sensitivity for $\Delta \omega_{L V}$ in the most advanced experiments 18, 19], this results in a stringent bound on $\left|N^{i}\right|$ :

$$
\kappa \sim \frac{e E p_{\text {nucl }}}{M m_{p}} \sim \frac{Z^{1 / 3} \mathrm{fm}^{-3} \alpha}{M m_{p}} \Rightarrow \frac{10^{19} \mathrm{GeV}}{M}\left|N^{i}\right|<10^{-9} .
$$

Here $m_{p}$ is the proton mass and $p_{\text {nucl }} \sim \mathrm{fm}^{-1}$ is the typical nucleon momentum. A more refined nuclear calculation can be done for mercury and xenon nuclei used in [18, 19] if needed.

\section{$L V$ precession of the angular momentum of a paramagnetic atom}

If for some unexpected reasons the effective electron LV coupling $b^{\mu}$ is close to zero, the interaction term proportional to $c^{\mu}$ in (56) would still induce a coupling of the electron angular momentum $j$ inside a paramagnetic atom to the spatial component of $N_{A}^{\mu} / 2-N^{\mu}$ with $\mathcal{H}_{\mathrm{eff}}=\kappa j_{i}\left(N_{A}^{i} / 2-N^{i}\right)$. In this case, the characteristic scale connecting $\Delta \omega_{L V}$ with $\mathrm{LV}$ parameters is the typical momentum of atomic electrons, $\mu \sim p_{\text {atomic }} \sim \alpha m_{e}$. Apart from an overall coefficient, the atomic matrix element responsible for this interaction has the same strength as the usual spin-orbit interaction, resulting in the estimate of $\kappa$

$$
\kappa \sim Z^{2} \alpha^{2} \frac{\alpha^{2} m_{e}^{2}}{M} \Rightarrow \frac{10^{19} \mathrm{GeV}}{M}\left|N^{i}-N_{A}^{i} / 2\right|<10^{-2} .
$$

\section{CPT-odd anomalous magnetic moment of electrons and positrons}

The limits explored so far do not use the fact that LV operators of dimension five break $\mathrm{CPT}$, whereas the experiments [18, 19] are done with normal matter. Some other experi- 
ments explicitly compare properties of electrons and positrons, and can therefore be used to constrain LV CPT-odd operators. For example, a $d^{0}$-proportional term in (56) induces an interaction between the electron spin with a magnetic field, and thus contributes to the anomalous magnetic moment of electrons and positrons. The different $g$-factors for electrons and positrons are limited at $10^{-12}$ level: $\left|g_{e}-g_{\bar{e}}\right|<8 \times 10^{-12}[\underline{51}]$.

The interaction Hamiltonian for electrons and positrons, corrected by the CPT-odd $d^{0}$ proportional interaction, takes the form:

$$
\mathcal{H}_{\mathrm{eff}}^{e}=-e d^{0} \frac{\vec{B} \cdot \vec{S}}{S}-|\mu| \frac{\vec{B} \cdot \vec{S}}{S}, \quad \mathcal{H}_{\mathrm{eff}}^{\bar{e}}=-e d^{0} \frac{\vec{B} \cdot \vec{S}}{S}+|\mu| \frac{\vec{B} \cdot \vec{S}}{S} .
$$

This gives a bound on the time-like component of $N_{V}^{\mu}$ :

$$
\frac{m_{e}}{M}\left|N_{V}^{0}\right|<2 \times 10^{-12} \quad \Rightarrow \quad \frac{10^{19} \mathrm{GeV}}{M}\left|N_{V}^{0}\right|<10^{10}
$$

Obviously, this limit is inferior to those derived from searches of the breakdown of rotational invariance [18, 19].

It is interesting to note that the CPT-violating correction to the magnetic moments of electrons and positrons arises in LV SQED even when SUSY is unbroken. At first sight this seems to be at odds with the Ferrara-Remiddi theorem which forbids emergence of the anomalous magnetic moment of the electron in the exact SUSY limit [52]: the anomalous magnetic moment of the electron, $e(4 m)^{-1} \bar{\Psi} \sigma_{\mu \nu} \Psi F^{\mu \nu}$, should appear as the highest component of some superfield, but no such supermultiplet exists [52]. However, one of the assumptions leading to this result is Lorentz invariance, therefore when SQED is extended by LV operators, the anomalous magnetic moment, $e M^{-1} N_{-}^{\mu} \bar{\Psi} \widetilde{F}_{\mu \nu} \gamma^{\nu} \gamma^{5} \Psi$, does arise as the highest component of a superfield operator, namely (91).

\section{Consequences for some dimension five operators in LV SQED}

The two most stringent limits, (57) and (58), are sensitive to different linear combinations of $N^{i}$ and $N_{A}^{i}$ vectors, thus imposing similar strength constraints on $N^{i}$ and $N_{A}^{i}$ separately. In order to impose a constraint on $N_{V}^{i}$, one has to make further assumptions about $\delta_{s}$. In the full MSSM scenario (as opposed to its SQED subset), parity is broken above the weak scale. Hence, a $\delta_{s}$ at a percent level or larger arises form radiative corrections even if the boundary conditions at $M$ are parity conserving, i.e. $m_{s}^{+}(M)=m_{s}^{-}(M)$. This provides a 
sensitivity to $N_{V}^{i}$ at the level of $10^{-10}$. The time-like components of vectors $N_{ \pm}^{\mu}$ and $N^{\mu}$ are also constrained: the motion of the earth and the solar system introduces a dependence of the laboratory frame on the velocity relative to the fixed vector backgrounds. Therefore, a non-zero $N^{0}$ would "mix" with $N^{i}$ at $O(v / c) \sim 10^{-3}-10^{-4}$ level. As a result, $10^{-6}-10^{-8}$ level constraints can be imposed on $N_{V}^{0}, N_{A}^{0}$ and $N^{0}$.

$\mathrm{LV}$ induced by $T^{\mu \nu \rho}$ (13) is also subject to experimental constraints. For example, a three dimensional vector $f^{k}=\epsilon_{i j k} T^{i 0 j}$, obtained from the tensor $T^{\mu \nu \rho}$, leads essentially to the same effects as vector $c^{k}$, and is therefore subject to bounds analogous to (58) and (59). Other components of $T^{\mu \nu \rho}$ can be limited using their contributions to $f^{k}$ caused by earth motion effects.

\section{Absence of Planck-scale bounds on dimension six LV operators}

Finally, we would like to assert that limits on dimension six operators are not able to rule out LV modifications at $M_{\mathrm{Pl}}^{-2}$ level. Many of the operators listed in (16) and (18) contain antisymmetric tensors. After the inclusion of SUSY breaking, such terms can mix with the $m_{e} \bar{\Psi} \sigma_{\mu \nu} \Psi$ operator, leading to LV spin precession of the electron. Assuming that the sizes of the dimensionless tensors in (16) and (18) are $O(1)$, one can estimate the sensitivity to $M$ via the dimension six LV operators: $M^{2} \sim m_{e} m_{s}^{2} /\left(10^{-28} \mathrm{GeV}\right)$. This translates into a bound of $M \sim 10^{14} \mathrm{GeV}$ for $m_{s} \sim 100 \mathrm{GeV}$, which is lower than the Planck scale. On the other hand, we notice that in the framework of the LV MSSM the sensitivity to $M$ via dimension six LV operators will be higher, when observables in the quark sector are employed. Indeed, we expect $m_{e}$ to be replaced by $m_{q}$, and $10^{-28} \mathrm{GeV}$ by $10^{-32} \mathrm{GeV}$, as experiments searching for anomalous spin precession of nucleons are intrinsically more precise. In this case the sensitivity to $M$ would get close to the Planck scale, and future increase of the experimental sensitivity may probe this type of models. Although undoubtedly very interesting, more detailed study of the observational consequences of dimension six LV operators goes beyond the scope of the present paper. 


\section{DISCUSSION AND CONCLUSIONS}

We have constructed a dimension five LV extension of SQED, as a subset of the full LV MSSM. The LV modifications are power-suppressed by the UV scale $M$ and decouple in the limit of $M \rightarrow \infty$. In the leading order in the inverse UV scale, $O\left(M^{-1}\right)$, dimension five LV operators can be coupled to two types of LV backgrounds. There are three background vectors $N^{\mu}, N_{+}^{\mu}$ and $N_{-}^{\mu}$, as well as an irreducible rank three tensor $T^{\mu \nu \lambda}$ (antisymmetric in $\nu \lambda$ ). The corresponding LV operators are all CPT-odd. At the dimension six level LV operators are CPT-even; their classification has been given in this paper.

We have explored quantum effects in the presence of the LV terms. We have shown that no $D$-term is induced and the anomaly cancellation condition is not altered by the presence of LV in the limit of exact SUSY. The RGE's for LV operators of dimension five were derived in the limits of exact and softly-broken SUSY. Once SUSY is broken, dimension three operators can be generated. The transmutation of dimension five LV operators into dimension three is controlled by the scale of soft SUSY breaking. This alleviates the LV naturalness problem, because the potentially problematic quadratic divergences are stabilized at the SUSY breakdown scale. In order to obtain phenomenologically applicable formulas, we broke SUSY by introducing scalar electron masses, and calculated the resulting effective LV Lagrangian for electrons. A dimension three operator for photons, the CS term, is not generated at the loop level. It is remarkable that none of the LV operators, considered in this paper, lead to high-energy modifications of dispersion relations. Therefore, none of the stringent astrophysics-derived limits on LV parameters [12, 13] apply to LV SQED.

We have obtained explicit component expressions for LV interactions generated by vector and tensor backgrounds, which allowed us to derive observational consequences of LV in SQED. Using the results of high-precision searches for LV spin interactions, we derived stringent limits on some linear combinations of LV parameters. The most stringent results resulted from a one-loop induced coupling between the electron axial vector current and some combination of the background vectors $N^{\mu}, N_{+}^{\mu}$ and $N_{-}^{\mu}$. The strongest bound was obtained from the absence of anomalous spin precession for electrons, which is checked at a level better than $10^{-28} \mathrm{GeV}$ by torsion balance experiments [50]. Assuming that the UV scale is of the same order of magnitude as the Planck scale, we were able to constrain one linear combination of $N^{i}, N_{+}^{i}$ and $N_{-}^{i}$ at the level better than $O\left(10^{-12}\right)$. Conversely, if we 
insist that $N^{i} \sim O(1)$, such experiments provide a sensitivity to the LV ultraviolet scale which is more than ten orders of magnitude larger than the Planck scale. Other precision experiments [18, 19] provide stringent constraints on different linear combinations of the LV vector and tensor backgrounds.

The existence of strong constraints on LV at dimension five level (with or without SUSY), poses a serious challenge for theories that predict $L V$ at $1 / M_{\mathrm{Pl}}$ level. Therefore, either such theories are ruled out, or they require abandoning an effective field theory description of LV. (The latter does not seem a reasonable alternative to us.) However, it might be that dimension five operators are forbidden by some additional symmetry reasons, such as e.g. CPT. At the next order, $O\left(M^{-2}\right)$, Planck suppressed LV effects are not excluded. (The best constraints may be better than the Planck scale [53], but are applicable only to operators that modify high-energy dispersion relations.) The classification of dimension six LV operators in SQED has shown that they couple to symmetric or antisymmetric two-index tensor backgrounds. Non of these operators lead to modifications of the dispersion relations hence the bounds of 53] do not apply. As we discussed at length for dimension five LV operators, similarly, some of dimension six operators will transmute into dimension four operators due to quantum effects in the presence of soft-breaking terms. The scale of the transmutation is controlled by the SUSY breakdown scale, which gives an estimate for the size of LV backgrounds at dimension four as $m_{s}^{2} / M^{2} \sim 10^{-32}$ for $m_{s} \sim 1 \mathrm{TeV}$ and $M \sim 10^{19}$ $\mathrm{GeV}$. This prediction comes close to the experimental sensitivity to such operators, and therefore deserves further study in the framework of LV MSSM.

\section{Acknowledgments}

SGN is indebted to M. Voloshin and M. Shifman for useful discussions about related field theoretical issues. SGN would like to thank the Perimeter Institute and the University of Guelph for their kind hospitality at various stages of this project. The work of P.B. and M.P. is supported in part by the N.S.E.R.C. of Canada. SGN has been supported in part by the Department of Energy under contract DE-FG02-94ER40823 at the University of Minnesota. 


\section{Appendix A: REDUCTION OF CHIRAL LV OPERATORS ON EQUATIONS OF MOTION}

The component expressions for LV terms in the chiral sector are given in (46). They can be transformed further by eliminating the auxiliary fields and higher derivatives via the equations of motion. Writing the result in terms of Dirac four-spinors, we get the following rather lengthy expression:

$$
\begin{aligned}
& \mathcal{L}_{\mathrm{LV}}^{\text {matter }}=-\frac{N_{A}^{\mu}}{M} \frac{1}{4} e \bar{\Psi} \epsilon_{\mu \nu \rho \sigma} F^{\rho \sigma} \gamma^{\nu} \Psi-\frac{N_{V}^{\mu}}{M} \frac{1}{4} e \bar{\Psi} \epsilon_{\mu \nu \rho \sigma} F^{\rho \sigma} \gamma^{\nu} \gamma^{5} \Psi+ \\
& +\frac{N_{+}^{\mu}}{M}\left[\frac{1}{2} i e \mathcal{D}^{\nu} \bar{z}_{+} \epsilon_{\mu \nu \rho \sigma} F^{\rho \sigma} z_{+}+\frac{1}{2} e\left(\bar{z}_{+} F_{\mu \nu} \mathcal{D}^{\nu} z_{+}+\mathcal{D}^{\nu} \bar{z}_{+} F_{\mu \nu} z_{+}\right)\right. \\
& \left.-\frac{i}{2} e^{2}\left(\mathcal{D}_{\mu} \bar{z}_{+} z_{+}-\bar{z}_{+} \mathcal{D}_{\mu} z_{+}\right)\left\{z_{-} \bar{z}_{-}-\bar{z}_{+} z_{+}\right\}\right]+ \\
& +\frac{N_{-}^{\mu}}{M}\left[-\frac{1}{2} i e z_{-} \epsilon_{\mu \nu \rho \sigma} F^{\rho \sigma} \mathcal{D}_{\nu} \bar{z}_{-}-\frac{1}{2} e\left(z_{-} F_{\mu \nu} \mathcal{D}^{\nu} \bar{z}_{-}+\mathcal{D}^{\nu} z_{-} F_{\mu \nu} \bar{z}_{-}\right)\right. \\
& \left.-\frac{i}{2} e^{2}\left(\mathcal{D}_{\mu} z_{-} \bar{z}_{-}-z_{-} \mathcal{D}_{\mu} \bar{z}_{-}\right)\left\{z_{-} \bar{z}_{-}-\bar{z}_{+} z_{+}\right\}\right]- \\
& -\frac{N_{+}^{\mu}}{M} e^{2} \bar{z}_{+} \bar{\lambda} \gamma^{\mu} \gamma^{5} \lambda z_{+}-\frac{N_{-}^{\mu}}{M} e^{2} z_{-} \bar{\lambda} \gamma^{\mu} \gamma^{5} \lambda \bar{z}_{-}- \\
& -\frac{N_{+\mu}}{M} \frac{\sqrt{2}}{2} e\left(\bar{\Psi} \gamma^{\nu} \gamma^{\mu} P_{R} \lambda \mathcal{D}_{\nu} z_{+}+\mathcal{D}_{\nu} \bar{z}_{+} \bar{\lambda} \gamma^{\mu} \gamma^{\nu} P_{L} \Psi\right)+ \\
& +\frac{N_{-\mu}}{M} \frac{\sqrt{2}}{2} e\left(\mathcal{D}_{\nu} z_{-} \bar{\lambda} \gamma^{\mu} \gamma^{\nu} P_{R} \Psi+\bar{\Psi} \gamma^{\nu} \gamma^{\mu} P_{L} \lambda \mathcal{D}_{\nu} \bar{z}_{-}\right)+ \\
& +\frac{N_{+}^{\mu}}{M} \frac{\sqrt{2}}{2} e\left(\bar{\Psi} P_{R} \mathcal{D}_{\mu} \lambda z_{+}+\bar{z}_{+} \mathcal{D}_{\mu} \bar{\lambda} P_{L} \Psi\right)- \\
& -\frac{N_{-}^{\mu}}{M} \frac{\sqrt{2}}{2} e\left(z_{-} \mathcal{D}_{\mu} \bar{\lambda} P_{R} \Psi+\bar{\Psi} P_{L} \mathcal{D}_{\mu} \lambda \bar{z}_{-}\right)+ \\
& +\frac{N_{A \mu}}{M} \frac{1}{2} e^{2} \bar{\Psi} \gamma^{\mu} \Psi\left\{z_{-} \bar{z}_{-}-\bar{z}_{+} z_{+}\right\}+ \\
& +\frac{N_{V \mu}}{M} \frac{1}{2} e^{2} \bar{\Psi} \gamma^{\mu} \gamma^{5} \Psi\left\{z_{-} \bar{z}_{-}-\bar{z}_{+} z_{+}\right\}- \\
& -\frac{N_{A \mu}}{M} \frac{\sqrt{2}}{2} i e\left(\bar{m}_{e} \bar{\Psi} \gamma^{\mu} P_{L} \lambda \bar{z}_{-}-m_{e} z_{-} \bar{\lambda} \gamma^{\mu} P_{L} \Psi\right)+ \\
& +\frac{N_{A \mu}}{M} \frac{\sqrt{2}}{2} i e\left(m_{e} \bar{\Psi} \gamma^{\mu} P_{R} \lambda z_{+}-\bar{m}_{e} \bar{z}_{+} \bar{\lambda} \gamma^{\mu} P_{R} \Psi\right)+ \\
& +\frac{N_{V \mu}}{M} 2 i m_{e} \bar{m}_{e}\left(\bar{z}_{+} \mathcal{D}_{\mu} z_{+}+z_{-} \mathcal{D}_{\mu} \bar{z}_{-}\right)+\frac{N_{V \mu}}{M} m_{e} \bar{m}_{e} \bar{\Psi} \gamma^{\mu} \Psi .
\end{aligned}
$$

The first, second, and last terms in (A1) enter the reduced Lagrangian (48) that involves only electrons, positrons and photons. The rather lengthy form of (A1) and the large number of diagrams that these interactions can create, underline the superiority of the superfield method over the component calculations for all processes with momenta larger than $m_{s}$. 


\section{Appendix B: CONVENTIONS AND NOTATIONS}

Our notations for the superfield formalism are based on Wess \& Bagger [29]. Covariant derivatives and hermitean conjugation are taken from [54] with a proper adaptation. We use the $(-+++)$ metric signature. All spinor algebra definitions can be found in [29], and we list here only some minor conventional departures. Unlike in [29], we denote the space-time Lorentz indices by letters from the middle of the Greek alphabet: $v_{\mu}, \sigma_{\nu}, N^{\rho}$, etc, as one is normally accustomed to in QFT. Spinor indices are taken, also as commonly accepted, from the beginning of the Greek alphabet: $\theta^{\alpha}, \epsilon_{\beta \gamma}, \bar{\psi}_{\dot{\delta}}$. Spinor derivatives are designated as

$$
\partial_{\alpha}=\frac{\partial}{\partial \theta^{\alpha}}, \quad \partial^{\alpha}=\epsilon^{\alpha \beta} \partial_{\beta}
$$

We use a notation with a slash in the case where a Lorentz vector is contracted with a $\sigma$-matrix, or a $\gamma$-matrix:

$$
\psi=v^{\mu} \sigma_{\mu}, \quad \bar{A}=A^{\mu} \bar{\sigma}_{\mu}, \quad \not h=n^{\mu} \gamma_{\mu} .
$$

For switching from Weyl to Dirac spinors we followed the notations of [55]. Weyl representation for Dirac spinors is the most appropriate in this case, where two Weyl spinors combine into one Dirac spinor:

$$
\Psi=\left(\begin{array}{c}
\xi_{\alpha} \\
\bar{\chi}^{\dot{\alpha}}
\end{array}\right), \quad \bar{\Psi}=\left(\begin{array}{c}
\chi^{\alpha} \\
\bar{\xi}_{\dot{\alpha}}
\end{array}\right),
$$

and the $\gamma$-matrices take the form

$$
\gamma^{\mu}=\left(\begin{array}{cc}
0 & \sigma^{\mu} \\
\bar{\sigma}^{\mu} & 0
\end{array}\right), \quad \gamma^{5}=\left(\begin{array}{cc}
1 & 0 \\
0 & -1
\end{array}\right)
$$

For complex conjugation, we use the notion of hermitean conjugation defined in [54]. When translated into the Wess \& Bagger notations, it implies

$$
\begin{array}{ll}
\left(\psi_{\alpha}\right)^{\dagger}=\bar{\psi}_{\dot{\alpha}}, & \left(\psi^{\alpha}\right)^{\dagger}=\bar{\psi}^{\dot{\alpha}}, \\
\partial_{\alpha}^{\dagger}=\bar{\partial}_{\dot{\alpha}}, & \partial_{\mu}^{\dagger}=-\partial_{\mu}, \\
D_{\alpha}^{\dagger}=-\bar{D}_{\dot{\alpha}}, & \left(\nabla_{\alpha}\right)^{\dagger}=-\bar{\nabla}_{\dot{\alpha}}, \\
W_{\alpha}^{\dagger}=\bar{W}_{\dot{\alpha}} . &
\end{array}
$$


Finally, the expansion of the chiral superfields of SQED in components is defined as

$$
\Phi_{ \pm}=z_{ \pm}+\sqrt{2} \theta \psi_{ \pm}+\theta^{2} F_{ \pm}
$$

while the vector superfield in the Wess-Zumino gauge is given by

$$
V=-\theta \sigma^{\mu} \bar{\theta} A_{\mu}+i \theta^{2} \bar{\theta} \bar{\lambda}-i \bar{\theta}^{2} \theta \lambda+\frac{1}{2} \theta^{2} \bar{\theta}^{2} D
$$

[1] D. Colladay and V. A. Kostelecky, Phys. Rev. D55, 6760 (1997), hep-ph/9703464.

[2] S. R. Coleman and S. L. Glashow, Phys. Rev. D59, 116008 (1999), hep-ph/9812418.

[3] T. Jacobson, S. Liberati, and D. Mattingly (2004), hep-ph/0407370.

[4] M. Pospelov and M. Romalis, Phys. Today 57N7, 40 (2004).

[5] G. Sigl (2004), hep-ph/0408165.

[6] D. Mattingly (2005), gr-qc/0502097.

[7] M. R. Douglas and N. A. Nekrasov, Rev. Mod. Phys. 73, 977 (2001), hep-th/0106048.

[8] L. Smolin (2003), hep-th/0303185.

[9] H. Vucetich (2005), gr-qc/0502093.

[10] S. M. Carroll, G. B. Field, and R. Jackiw, Phys. Rev. D41, 1231 (1990).

[11] G. Amelino-Camelia, J. R. Ellis, N. E. Mavromatos, D. V. Nanopoulos, and S. Sarkar, Nature 393, 763 (1998), astro-ph/9712103.

[12] T. Jacobson, S. Liberati, and D. Mattingly, Nature 424, 1019 (2003), astro-ph/0212190.

[13] R. J. Gleiser and C. N. Kozameh, Phys. Rev. D64, 083007 (2001), gr-qc/0102093.

[14] V. A. Kostelecky and M. Mewes, Phys. Rev. Lett. 87, 251304 (2001), hep-ph/0111026.

[15] O. Bertolami and C. S. Carvalho, Phys. Rev. D61, 103002 (2000), gr-qc/9912117.

[16] S. Sarkar, Mod. Phys. Lett. A17, 1025 (2002), gr-qc/0204092.

[17] O. Bertolami, R. Lehnert, R. Potting, and A. Ribeiro, Phys. Rev. D69, 083513 (2004), astro$\mathrm{ph} / 0310344$.

[18] C. Berglund et al., Phys. Rev. Lett. 75, 1879 (1995), physics/0007049.

[19] D. Bear, R. E. Stoner, R. L. Walsworth, V. A. Kostelecky, and C. D. Lane, Phys. Rev. Lett. 85, 5038 (2000), physics/0007049.

[20] D. Sudarsky, L. Urrutia, and H. Vucetich, Phys. Rev. Lett. 89, 231301 (2002), gr-qc/0204027. 
[21] R. C. Myers and M. Pospelov, Phys. Rev. Lett. 90, 211601 (2003), hep-ph/0301124 and gr-qc/0402028.

[22] V. A. Kostelecky and S. Samuel, Phys. Rev. D40, 1886 (1989).

[23] S. Groot Nibbelink and M. Pospelov, Phys. Rev. Lett. 94, 081601 (2005), hep-ph/0404271.

[24] I. Mocioiu, M. Pospelov, and R. Roiban, Phys. Lett. B489, 390 (2000), hep-ph/0005191.

[25] A. Anisimov, T. Banks, M. Dine, and M. Graesser, Phys. Rev. D65, 085032 (2002), hep$\mathrm{ph} / 0106356$.

[26] I. Mocioiu, M. Pospelov, and R. Roiban, Phys. Rev. D65, 107702 (2002), hep-ph/0108136.

[27] C. E. Carlson, C. D. Carone, and R. F. Lebed, Phys. Lett. B549, 337 (2002), hep-ph/0209077.

[28] P. Jain and J. P. Ralston (2005), hep-ph/0502106.

[29] J. Wess and J. Bagger, Supersymmetry and supergravity, princeton, USA: Univ. Pr. (1992) 259 p.

[30] W. G. Ney, J. A. Helayel-Neto, and W. Spalenza (2004), hep-th/0410287.

[31] W. G. Ney, J. A. Helayel-Neto, and W. Spalenza, Proc. Sci. WC2004, 046 (2004), hepth/0502233.

[32] W. G. Ney, J. A. Helayel-Neto, and W. Spalenza (2005), hep-th/0502037.

[33] M. S. Berger and V. A. Kostelecky, Phys. Rev. D65, 091701 (2002), hep-th/0112243.

[34] M. S. Berger, Phys. Rev. D68, 115005 (2003), hep-th/0308036.

[35] V. Putz and R. Wulkenhaar, Int. J. Mod. Phys. A18, 3325 (2003), hep-th/0205094.

[36] D. Mikulovic, JHEP 01, 063 (2004), hep-th/0310065.

[37] H. Belich, J. L. Boldo, L. P. Colatto, J. A. Helayel-Neto, and A. L. M. A. Nogueira, Phys. Rev. D68, 065030 (2003), hep-th/0304166.

[38] H. Belich, M. A. De Andrade, and M. A. Santos (2005), hep-th/0503145.

[39] W. Fischler, H. P. Nilles, J. Polchinski, S. Raby, and L. Susskind, Phys. Rev. Lett. 47, 757 (1981).

[40] T. Hayashi, Y. Ohshima, K. Okuyama, and H. Suzuki, Prog. Theor. Phys. 100, 627 (1998), hep-th/9801062.

[41] J. Gates, S. James, M. T. Grisaru, M. E. Knutt, S. Penati, and H. Suzuki, Nucl. Phys. B596, 315 (2001), hep-th/0009192.

[42] K. Fujikawa, Phys. Rev. D29, 285 (1984).

[43] K.-i. Konishi and K.-i. Shizuya, Nuovo Cim. A90, 111 (1985). 
[44] S. Cecotti, S. Ferrara, and M. Villasante, Int. J. Mod. Phys. A2, 1839 (1987).

[45] R. Jackiw and V. A. Kostelecky, Phys. Rev. Lett. 82, 3572 (1999), hep-ph/9901358.

[46] J. M. Chung and P. Oh, Phys. Rev. D60, 067702 (1999), hep-th/9812132.

[47] A. A. Andrianov, P. Giacconi, and R. Soldati, JHEP 02, 030 (2002), hep-th/0110279.

[48] M. Perez-Victoria, JHEP 04, 032 (2001), hep-th/0102021.

[49] D. Colladay and V. A. Kostelecky, Phys. Rev. D58, 116002 (1998), hep-ph/9809521.

[50] B. R. Heckel, E. G. Adelberger, J. H. Gundlach, M. G. Harris, and H. E. Swanson, Torsion balance test of spin coupled forces, prepared for International Conference on Orbis Scientiae 1999: Quantum Gravity, Generalized Theory of Gravitation and Superstring Theory Based Unification (28th Conference on High-Energy Physics and Cosmology Since 1964), Coral Gables, Florida, 16-19 Dec 1999.

[51] R. K. Mittleman, I. I. Ioannou, H. G. Dehmelt, and N. Russell, Phys. Rev. Lett. 83, 2116 (1999).

[52] S. Ferrara and E. Remiddi, Phys. Lett. B53, 347 (1974).

[53] O. Gagnon and G. D. Moore, Phys. Rev. D70, 065002 (2004), hep-ph/0404196.

[54] S. J. Gates, M. T. Grisaru, M. Rocek, and W. Siegel, Front. Phys. 58, 1 (1983), hepth/0108200.

[55] S. P. Martin (1997), hep-ph/9709356.

[56] We would like to thank N. Arkani-Hamed for this comment. 